\title{
ACTIVE SUSPENSION CONTROL WITH DIRECT-DRIVE TUBULAR LINEAR BRUSHLESS PERMANENT-MAGNET MOTOR
}

\author{
A Thesis \\ by \\ SEUNGHO LEE \\ Submitted to the Office of Graduate Studies of \\ Texas A\&M University \\ in partial fulfillment of the requirements for the degree of \\ MASTER OF SCIENCE
}

May 2009

Major Subject: Mechanical Engineering 


\title{
ACTIVE SUSPENSION CONTROL WITH DIRECT-DRIVE TUBULAR LINEAR BRUSHLESS PERMANENT-MAGNET MOTOR
}

\author{
A Thesis
}

by

\section{SEUNGHO LEE}

\section{Submitted to the Office of Graduate Studies of Texas A\&M University in partial fulfillment of the requirements for the degree of \\ MASTER OF SCIENCE}

Approved by:

Chair of Committee, Won-Jong Kim

Committee Members, Bryan Rasmussen James Boyd

Head of Department, Dennis O’Neal

May 2009

Major Subject: Mechanical Engineering 


\begin{abstract}
Active Suspension Control with Direct-Drive Tubular Linear Brushless PermanentMagnet Motor. (May 2009)

Seungho Lee, B.S., Yonsei University

Chair of Advisory Committee: Dr. Won-Jong Kim
\end{abstract}

Recently, active suspension has been applied to many commercial automobiles. To develop the control algorithm for active suspension, a quarter-car test bed was built by using a direct-drive tubular linear brushless permanent-magnet motor (LBPMM) as a force-generating component. Two accelerometers and a linear variable differential transformer (LVDT) are used in this quarter-car test bed. Three pulse-width-modulation (PWM) amplifiers supply the currents in three phases. Simulated road disturbance is generated by a rotating cam. Modified lead-lag control, linear-quadratic (LQ) servo control with a Kalman filter, and the fuzzy control methodologies were implemented for active-suspension control. In the case of fuzzy control, asymmetric membership functions were introduced. This controller could attenuate road disturbance by up to $78 \%$. Additionally, a sliding-mode controller (SMC) is developed with a different approach from the other three control methodologies. While SMC is developed for the position control, the other three controllers are developed for the velocity control. SMC showed inferior performance due to the drawback of the implemented chattering-proof method. 
Both simulation and experimental results are presented to demonstrate the effectiveness of these four control methodologies. 


\section{ACKNOWLEDGEMENTS}

First of all, I would like to thank my committee chair, Dr. Won-Jong Kim, and my committee members, Dr. Bryan Rasmussen, and Dr. James Boyd, for their guidance and support throughout this research.

Thanks also go to my friends and colleagues and the department faculty and staff for making my time at Texas A\&M University a great experience. Especially I want to extend my gratitude to the former students, Mr. Bryan Murphy, who developed the LBPMM, and Mr. Justin Allen, who designed the quarter-cat test bed. Their research was a foundation for my research.

Finally, thanks to my family for their encouragement and love. 


\section{TABLE OF CONTENTS}

Page

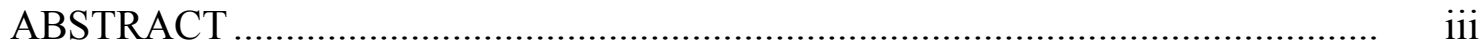

ACKNOWLEDGEMENTS …...........................................................

TABLE OF CONTENTS ....................................................................... vi

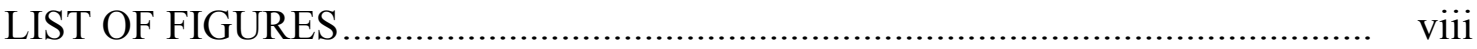

LIST OF TABLES …............................................................................

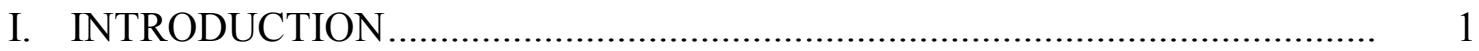

II. TEST BED FOR ACTIVE SUSPENSION CONTROL ................................ 6

A. Linear Brushless Permanent-Magnet Motor .......................................... 6

B. Quarter-Car Test Bed..................................................................... 7

1) Wheel .............................................................................. 11

2) Accelerometer .................................................................. 12

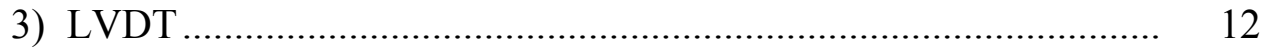

4) PWM amplifier .................................................................... 13

5) Conditioning circuit ............................................................ 13

6) dSPACE 1104 control board ............................................... 14

7) Disturbance generating system ......................................... 15

III. CONTROL METHODOLOGIES AND EXPERIMENTAL RESULTS .......... 17

A. Modified Lead-Lag Control .......................................................... 17

B. Linear-Quadratic Servo Control ........................................................ 21

1) Kalman filter design .............................................................. 26

C. Fuzzy Control ................................................................................. 29

D. Sliding-Mode Control......................................................................... 35

IV. PERFORMANCE COMPARISONS ....................................................... 44

V. CONCLUSIONS ................................................................................. 48 
Page

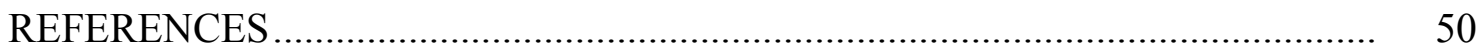

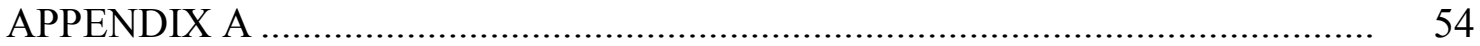

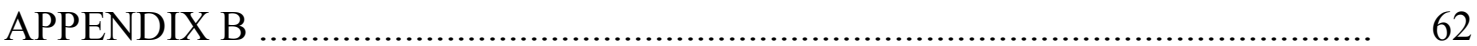

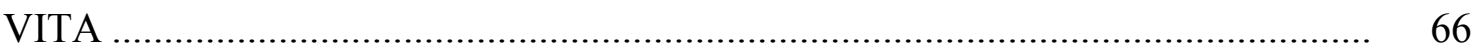




\section{LIST OF FIGURES}

Page

Figure 1 Schematic diagram of the LBPMM ......................................... 6

Figure 2 Photograph of the quarter-car test bed with active suspension ........ 8

Figure 3 Schematic diagram of the quarter-car test bed with active suspension

Figure 4 Free-body diagram of (a) the sprung mass and (b) the unsprung mass.....

Figure 5 Schematic diagram of the control architecture.....

Figure 6 Schematic diagram of the conditioning circuit.

Figure 7 Photograph of the disturbance-generating system.

Figure 8 Quarter-car and loop-transfer-function frequency responses of the quarter-car dynamics and the modified lead-lag controller.

Figure 9 Current flow of the modified lead-lag control in experiment for the 3.5- $\mathrm{Hz}$ disturbance

Figure 10 Experiment and simulation results of the modified lead-lag control for the $3.5-\mathrm{Hz}$ disturbance

Figure 11 Block diagrams of (a) the standard LQ servo control and (b) the LQ servo control with an integrator

Figure 12 Frequency responses of the loop transfer functions in the LQ servo control.

Figure 13 Estimated state comparison between simulation and experiment results

Figure 14 Experiment and simulation results of the LQ servo control 
Figure 15 (a) Membership functions for fuzzification. (b) Membership functions for defuzzification

Figure 16 Input-output relation of the asymmetric fuzzy controller

Figure 17 Experiment and simulation results of the fuzzy control

Figure 18 Generated force from the asymmetric membership functions for $-0.1 \mathrm{~m} / \mathrm{s}$ error

Figure 19 Generated force from the asymmetric membership functions for $0.1 \mathrm{~m} / \mathrm{s}$ error

Figure 20 SMC result in case of (a) $\varepsilon=0.002$, (b) $\varepsilon=0.004$, and (c) $\varepsilon=0.006$, respectively

Figure 21 SMC result in case of (a) $\varepsilon=0.007$, and (b) $\varepsilon=0.008$, respectively. 39

Figure 22 SMC result in case of $\varepsilon=0.009$ 40

Figure 23 State trajectory and sliding surface

Figure 24 Experiment and simulation results of the SMC with $\varepsilon=0.004$. 42

Figure 25 Disturbance attenuation performance of the modified lead-lag controller

Figure 26 Disturbance attenuation performance of the LQ servo controller 45

Figure 27 Disturbance attenuation performance of the fuzzy controller..... 46

Figure 28 Disturbance attenuation performance of the SMC 


\section{LIST OF TABLES}

Page

Table 1 Parameters and corresponding values of quarter-car model ............ 10

Table $2 \quad$ PWM amplifier gains ........................................................... 13

Table 3 Bolt winding and generated disturbance frequencies...................... 16

Table $4 \quad$ Rules of the fuzzy controller .......................................................... 31 


\section{INTRODUCTION}

Active suspension supports a vehicle and isolates passengers from road disturbances for ride quality and vehicle handling using force-generating components under feedback control. Notwithstanding its complexity, high cost, and power requirements, active suspension has been used by the luxury car manufactures, such as BMW, Mercedes-Benz, and Volvo. Development of an active-suspension system should be accompanied by the methodologies to control it. Considering costly commercial vehicles with active suspension, Allen constructed a quarter-car test bed to develop the control algorithms [1].

Many researchers developed active-suspension control techniques [2]-[10]. These researches could be categorized according to the applied control theories. When it comes to the LQ control, Peng, et al. presented the virtual input signal determined by the LQ optimal theory for active-suspension control [2]. Tang and Zhang applied linearquadratic-Gaussian (LQG) control, neural networks, and genetic algorithms in an active suspension and presented simulation results [3]. Sam, et al. applied LQ control to simulate an active-suspension system [4]. As for the robust control, Lauwerys, et al. developed a linear robust controller based on the $\mu$-synthesis for the active suspension of a quarter car [5]. Wang, et al. presented the algorithm to reduce the order of the $H_{\infty}$ controller in the application of active suspension [6].

This thesis follows the style of IEEE Transactions on Control Systems Technology. 
They were able to reduce the controller's order by nearly one third while the performance was only slightly degraded. Abbas, et al. applied sliding-mode control for nonlinear full-vehicle active suspension [7]. They considered not only the dynamics of the nonlinear full-vehicle active-suspension system but also the dynamics of the four actuators. Many neural-network controllers were also applied to active suspension. Jin, et al. developed a novel neural control strategy for an active suspension system [8]. By combining the integrated error approach with the traditional neural control, they were able to develop a simple-structure neural controller with small computational requirements, beneficial to real-time control. Kou and Fang established active suspension with an electro-hydrostatic actuator (EHA) and implemented a fuzzy controller [9]. They could attenuate the suspension deflection by $26.76 \%$ compared with passive suspension. Alleyne and Hedrick developed a nonlinear adaptive controller for active suspension with an electro-hydraulic actuator [10]. They analyzed a standard parameter adaptation scheme based on the Lyapunov analysis and presented a modified adaptation scheme for active suspension.

Several researchers used electro-hydraulic actuators for active suspension $[9,10]$. Electro-hydraulic actuators are powerful and less bulky compared to DC and AC actuators. Moreover, they can provide the skyhook damping effect, an ideal design of suspension [11]. However, electro-hydraulic actuators are highly nonlinear because of their hydraulic components such as a servo valve. In most studies, it was assumed that the chamber volume of the hydraulic actuator was constant while in fact the volume varied with the piston motion. This induced an additional uncertainty to the model. 
However, due to the compact design, an LBPMM has less modeling uncertainty and nonlinearity. Moreover, this LBPMM is directly applicable to active suspension without converting rotary motion to linear motion [12]. Besides its smooth, precise translational motion without cogging, the fact that the length of the mover can be conveniently adjusted makes it appropriate for the force-generating component in an active-suspension system. Other actuators such as an oleo-pneumatic unit [13] and a 3degree-of-freedom (3-DOF) vibration-isolation system [14] were also used for active suspension. The drawbacks of these actuators are bulkiness and design complexity. The oleo-pneumatic unit required a sealing structure. The 3-DOF vibration-isolation system consisted of five tables, magnets, springs, dampers, which led to a large size.

Realistic models of the car were considered in several research projects. Gao, et al. proposed a load-dependent controller for active suspension control [15]. They considered the sprung mass of the car varied with the load condition and assumed this value was measurable online. With this information they developed a much less conservative controller compared to a previous robust-control approach. Yagiz, et al. considered not only vertical but also pitch and roll motions of a nonlinear 7-DOF vehicle model [16]. They developed a SMC for the active suspension control in a full vehicle.

There are issues related with the limitations of active suspension and solutions. For example, Suda and Shiba proposed the energy regeneration in active suspension to solve the energy problem [17]. They proposed an energy regenerative damper system that converts vibration energy into useful energy. Then converted energy is used to the active suspension. 
When it comes to advanced control techniques, Concha and Cipriano developed a novel controller combined with the fuzzy and LQR controllers [18]. Gobbi, et al. proposed a new control method based on a stochastic optimization theory assuming that the road irregularity is a Gaussian random process and modeled an exponential power spectral density [19].

Since a human body is most susceptible to vibration at around $3 \mathrm{~Hz}(20 \mathrm{rad} / \mathrm{s})$ [20], disturbance from the road is modeled as a sinusoidal input with a frequency of 3.5 $\mathrm{Hz}(22 \mathrm{rad} / \mathrm{s})$ and a magnitude of $\pm 0.03 \mathrm{~m}$ in this research. The LBPMM was designed to be able to generate the force up to $29.6 \mathrm{~N}$ with a \pm 6 -A phase current [12]. Since $\mathrm{NdFeB}$ magnet would lose magnetization around $150^{\circ} \mathrm{C}$, control performance is compromised with the maximum current swing that yields temperature rise. As a result, controllers are designed to have the current limit of around $\pm 4 \mathrm{~A}$. The piezoelectric accelerometers (Piezotronics model 336B18) used in the quarter-car test bed also limit the performance. These accelerometers can be used only in the frequency range of 0.5 to $3000 \mathrm{~Hz}$ (3 to $20000 \mathrm{rad} / \mathrm{s}$ ). Particularly, this implies that our active-suspension system is not able to attenuate the disturbance with a frequency component lower than $0.5 \mathrm{~Hz}$.

The fact that the LBPMM is used for active suspension and four different classes of control methodologies are developed and successfully implemented is the key contribution of this research and differentiates this thesis from others. Especially, in the case of fuzzy control, an asymmetric fuzzy controller was implemented to compensate for DC offset in sensor data. As for the control methodologies, a modified lead-lag control was chosen to be developed first among the classical control methodologies. The 
LQ servo control was employed among the modern state-space-based control techniques. Then a fuzzy control was selected because it has infinitely many degrees of freedom to its design, and the information obtained from the previous two controllers could facilitate the determination of its design parameters. Aside from these three controllers, a SMC is lastly developed. While the previous three controllers are designed for the velocity control, this SMC is designed for the position control of the sprung mass.

This thesis is organized as follow. In Section II, components of the quarter-car test bed and its modeling are introduced. Since the LBPMM is the most important component, its working principle is summarized in Section II.A. Section II.B presents not only the modeling of the quarter-car test bed but also its major components such as wheel, accelerometer, LVDT, PWM amplifier, conditioning circuit, dSPACE 1104 control board, and disturbance-generating components. In Section III, control methodologies for the active suspension are presented. In Section III.A, implementation of a modified lead-lag controller and its disturbance attenuation performance are presented. Section III.B describes the design and performance of an LQ servo controller and the state estimation by a Kalman filter. Section III.C presents a fuzzy controller with asymmetric membership functions and its performance. Section III.D presents the design and the performance of a SMC. The disturbance attenuation performances of the four controllers designed in Section III are discussed in Section IV. The conclusions follow in Section V. 


\section{TEST BED FOR ACTIVE SUSPENSION CONTROL}

\section{A. Linear Brushless Permanent-Magnet Motor}

Figure 1 shows a conceptual configuration of the LBPMM that Murphy constructed [12]. The mover of the LBPMM consists of a series of cylindrical permanent magnets. The magnets are fixed in a brass tube and connected each other in an NS-NS-SN-SN fashion with spacers between the pairs. The stator consists of 9 coils ( 3 per each phase). The three-phase coils are represented by $A, B$, and $C$ in balanced three-phase operation. The magnets are aligned with the arrow pointing to the $\mathrm{N}$ pole. The pitch of these magnets is kept the same as that of the coils.

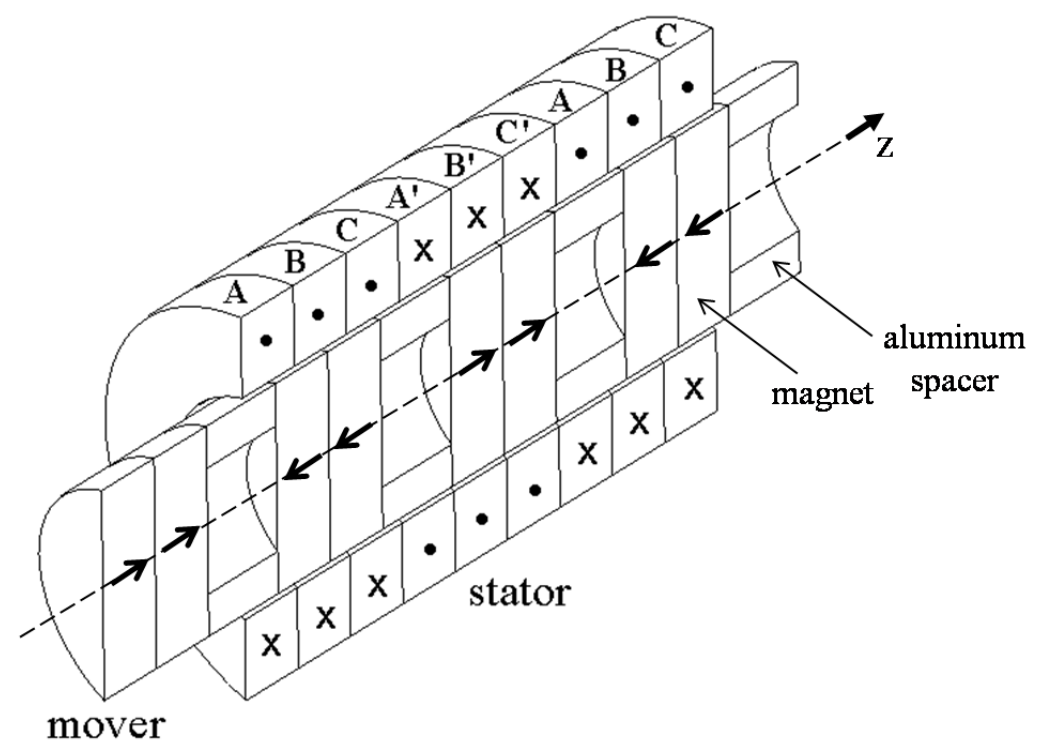

Figure 1. Schematic diagram of the LBPMM. The direction of the generated force on the mover is in the negative $z$-direction in this particular current distribution [12]. 
By the Lorentz force equation, the generated force is the vector cross product of the current density $\boldsymbol{J}$ in the coils and the magnetic flux density $\boldsymbol{B}$ generated by the magnets, $\boldsymbol{F}=\boldsymbol{J} \times \boldsymbol{B}$ [12]. The inverse Blondel-Park transformation in the LBPMM that governs the relationship between the three-phase currents and the desired force is defined as follows [12].

$$
\left[\begin{array}{l}
i_{a}(t) \\
i_{b}(t) \\
i_{c}(t)
\end{array}\right]=C\left[\begin{array}{cc}
2 & 0 \\
1 & \sqrt{3} \\
-1 & \sqrt{3}
\end{array}\right]\left[\begin{array}{l}
\cos \gamma_{1} z_{o} \\
\sin \gamma_{1} z_{0}
\end{array}\right] f_{z d}(t)
$$

where $i_{a}(t), i_{b}(t)$, and $i_{c}(t)$ are the currents flowing in phases $A, B$, and $C$, respectively. $f_{z d}$ is the desired force in the axial direction. $\gamma_{1}=|2 \pi / l|$, where $l$ is the pitch of the motor $(63.3 \mathrm{~mm}) . z_{0}$ is relative displacement between the mover and the stator. In active suspension, it is the distance between the sprung and unsprung masses. The inverse force constant $C$ was determined as $0.1383 \mathrm{~A} / \mathrm{N}$ by experiments [12].

\section{B. Quarter-Car Test Bed}

Figure 2 shows a photograph of the quarter-car test bed that Allen designed and constructed [1]. The sprung mass $\left(M_{s}\right)$ is considered to be the body of a car, and the unsprung mass $\left(M_{u s}\right)$ represents the mass between its suspension and a wheel. As shown in Figure 3, two masses are connected with a mechanical spring and the LBPMM. The stator of the LBPMM is fixed to the sprung mass and one end of the mover is fixed to the unsprung mass so that the force generated by the LBPMM can act on this quarter- 
car test bed. The rotating cam shown at the bottom of Figure 2 simulates sinusoidal road disturbance at various frequencies.

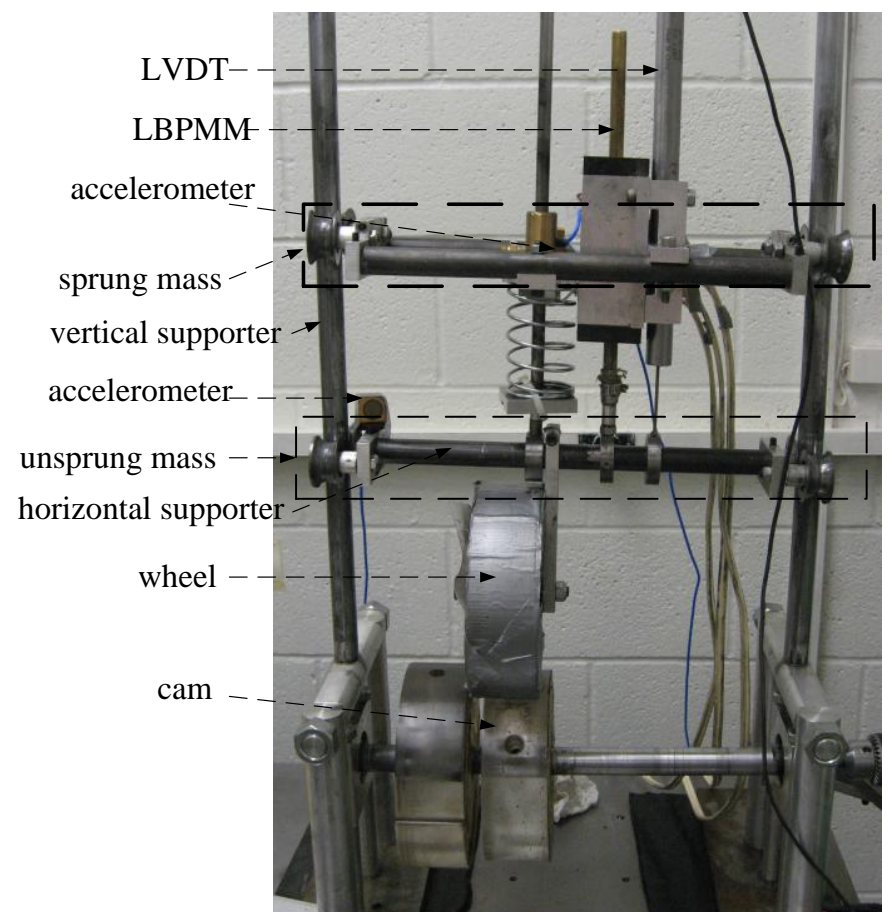

Figure 2. Photograph of the quarter-car test bed with active suspension [1].

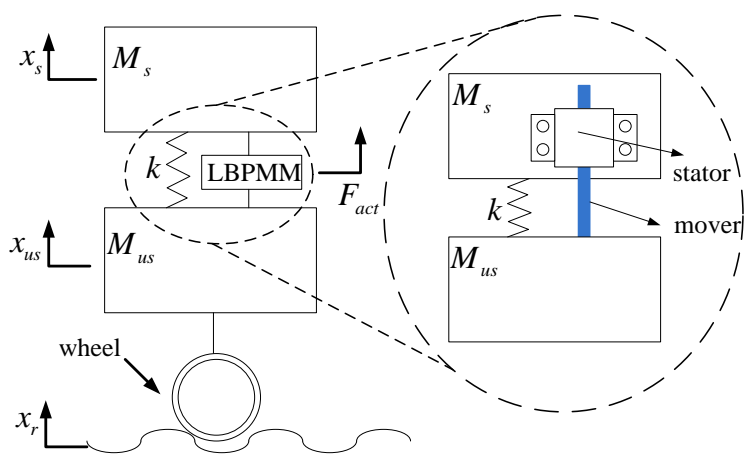

Figure 3. Schematic diagram of the quarter-car test bed with active suspension. 


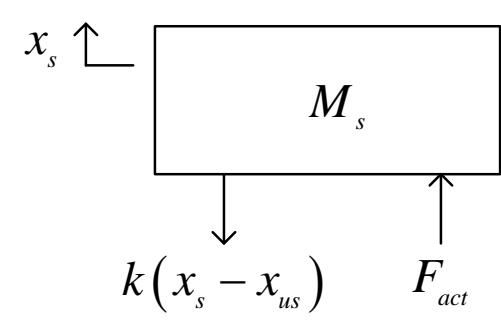

(a)

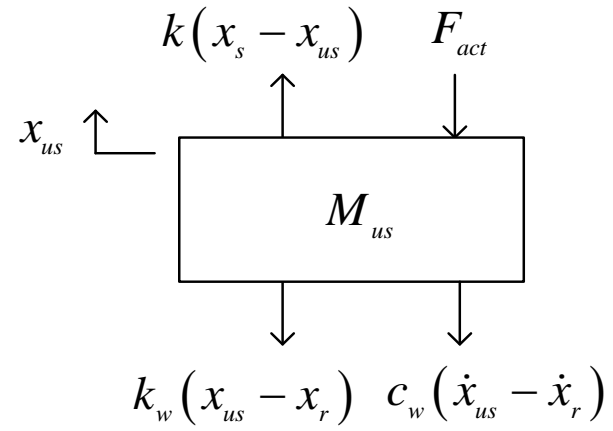

(b)

Figure 4. Free-body diagram of (a) the sprung mass and (b) the unsprung mass.

Figure 4 shows the free-body diagrams for the sprung mass and the unsprung mass of the quarter-car test bed. From the Figure 4 (a) and (b), equation (2) and (3) are obtained, respectively.

$$
\begin{gathered}
M_{s} \ddot{x}_{s}=-k\left(x_{s}-x_{u s}\right)+F_{a c t} \\
M_{u s} \ddot{x}_{u s}=k\left(x_{s}-x_{u s}\right)-F_{a c t}-k_{w}\left(x_{u s}-x_{r}\right)-c_{w}\left(\dot{x}_{u s}-\dot{x}_{r}\right),
\end{gathered}
$$

where $x_{s}(t)$ and $x_{u s}(t)$ are the positions of the sprung and unsprung masses, respectively, $x_{r}(t)$ is the sinusoidal disturbance generated by the rotating cam, and $F_{a c t}$ is the force generated by the LBPMM. Additionally, the wheel is modeled by the spring constant $k_{w}$ and the viscous damping coefficient $c_{w}$. The parameter values are given in Table 1 .

The equation of motion of the sprung mass is presented in (2). This motion is determined by the spring force proportional to its deflection by a spring constant $k$ and an actuation force generated by the LBPMM. The equation of motion of the unsprung 
mass is presented in (3). This motion is governed by not only the spring force and actuation force but also the forces imposed through the wheel. The LBPMM generates $F_{a c t}$ to mitigate the effect from the force $k\left(x_{s}-x_{u s}\right)$ on the motion of the sprung mass

Table 1

Parameters and corresponding values of quarter-car model

\begin{tabular}{ll}
\hline \hline Parameters & Values \\
\hline$M_{s}$ & $2.299 \mathrm{~kg}$ \\
$M_{u s}$ & $2.278 \mathrm{~kg}$ \\
$k$ & $1521 \mathrm{~N} / \mathrm{m}$ \\
$c_{w}$ & $50 \mathrm{~N}-\mathrm{s} / \mathrm{m}$ \\
$k_{w}$ & $156 \mathrm{~N} / \mathrm{m}$ \\
\hline \hline
\end{tabular}

Equations (2) and (3) are presented as a state-space form in (4). As in [11], the states of the quarter-car test bed are defined as $\left[\dot{x}_{s}(t) \quad \dot{x}_{u s}(t) \quad x_{s}(t)-x_{u s}(t) \quad x_{u s}(t)-x_{r}(t)\right]^{T}$ and its dynamics is expressed as the following state-space matrix form.

$$
\left[\begin{array}{c}
\ddot{x}_{s}(t) \\
\ddot{x}_{u s}(t) \\
\dot{x}_{s}(t)-\dot{x}_{u s}(t) \\
\dot{x}_{u s}(t)-\dot{x}_{r}(t)
\end{array}\right]=\left[\begin{array}{cccc}
0 & 0 & \frac{-k}{M_{s}} & 0 \\
0 & \frac{-c_{w}}{M_{u s}} & \frac{k}{M_{u s}} & \frac{-k_{w}}{M_{u s}} \\
1 & -1 & 0 & 0 \\
0 & 1 & 0 & 0
\end{array}\right]\left[\begin{array}{c}
\dot{x}_{s}(t) \\
\dot{x}_{u s}(t) \\
x_{s}(t)-x_{u s}(t) \\
x_{u s}(t)-x_{r}(t)
\end{array}\right]+\left[\begin{array}{c}
\frac{1}{M_{s}} \\
\frac{-1}{M_{u s}} \\
0 \\
0
\end{array}\right] F_{a c t}(t)+\left[\begin{array}{c}
0 \\
0 \\
0 \\
-1
\end{array}\right] \dot{x}_{r}(t)
$$

Figure 5 shows a schematic diagram of the control architecture. Accelerometers and the LVDT generate the sensor signals. Analog-to-digital (A/D) channels on the 
dSPACE 1104 control board receive these sensor signals. Controllers are implemented on this board and use the sensor signals for the active suspension control.

Since the A/D channels of the dSPACE 1104 board have an input voltage swing of $\pm 10 \mathrm{~V}$ and the output swing of the LVDT is $0 \mathrm{~V}$ to $5 \mathrm{~V}$, a conditioning circuit is used to shift the output range of the LVDT to match the input range of the A/D channels. Three PWM amplifiers are used to power the three-phase coils.

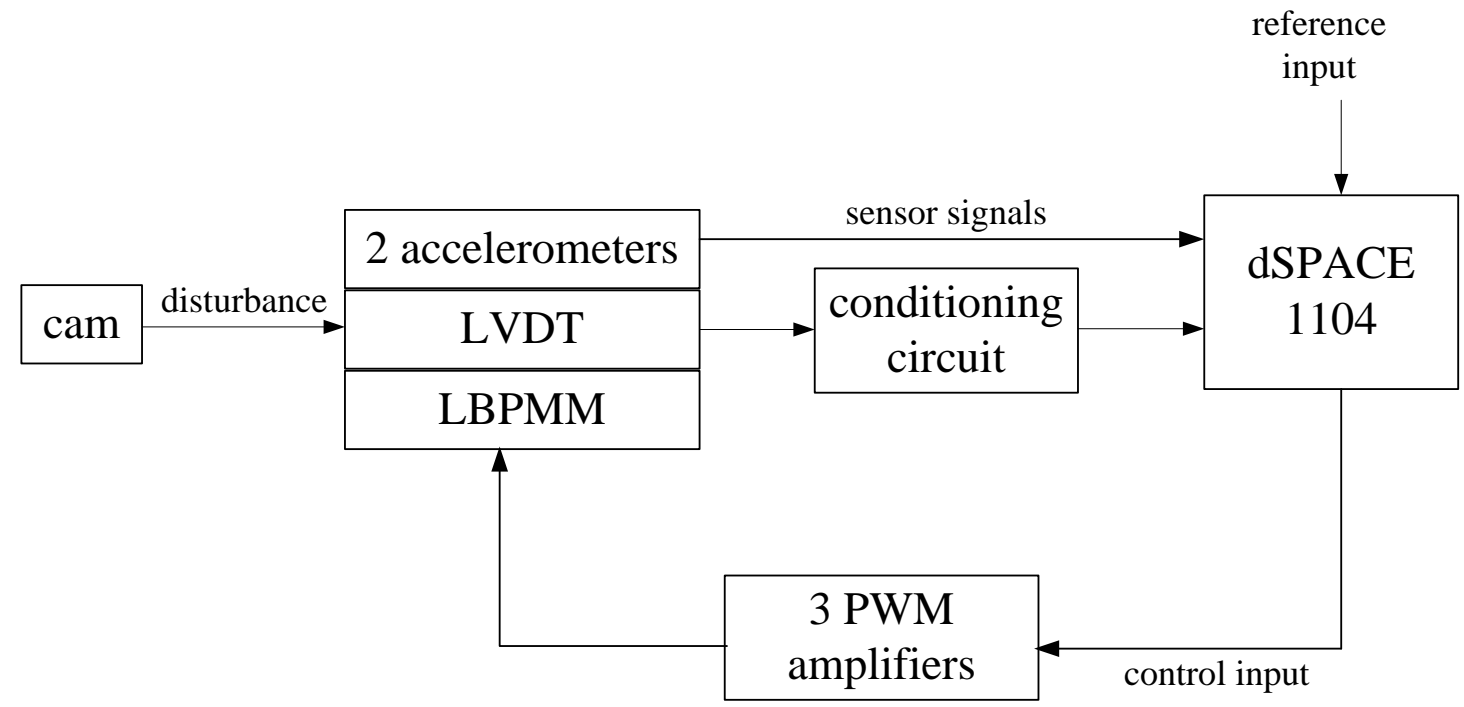

Figure 5. Schematic diagram of the control architecture.

1) Wheel

The wheel of the quarter-car test bed is covered with a tire. It was known that this tire is made of nature isoprene, which has Young's modulus $E$, from 0.0007 to 0.004 GPa [21]. A tape is used to glue the tire and wheel body together. Since the exact model of this wheel is very hard to obtain, the spring constant and the damping constant of this wheel are adjusted from the previous research [1] and determined. The adjustment of 
those constants is performed under the open-loop condition until the simulation and experimental results (Figure 10 when the controller is off) are coincided. Finally, spring constant of the wheel, $k_{w}$ and the damping coefficient, $c_{w}$ are determined as $156 \mathrm{~N} / \mathrm{m}$ and $50 \mathrm{~N}-\mathrm{s} / \mathrm{m}$, respectively.

\section{2) Accelerometer}

The accelerations of the sprung mass and unsprung mass are observed by the piezoelectric accelerometers (Piezotronics model 336B18). When the piezoelectric material undergoes deformation, a small voltage differential output is produced. Accordingly, the accelerometer creates a signal proportional to the acceleration of the device structure. The accelerometers have a frequency range of $0.5-3000 \mathrm{~Hz}$ with a gain factor of $10.28 \mathrm{mV} / \mathrm{g}$. The outputs of these accelerometers go to the signal conditioner (PCB model 482A22). This signal conditioner provides power to the accelerometers and produces the proportional voltage output with the range of $\pm 10 \mathrm{~V}$ according to its inputs.

\section{3) $L V D T$}

An LVDT (Schaevitz DC-SE 4000) is placed between the sprung mass and the unsprung mass. This LVDT directly measures the distance between those masses, which is the third state in the state-space representation, and is used in the case of the LQ servo control. The LVDT requires a 10-V input supplied by an Agilent 3644A power supply. The output of the LVDT is a linear voltage signal between $0-5 \mathrm{~V}$ with a maximum stroke length of $\pm 100 \mathrm{~mm}$. 


\section{4) PWM amplifier}

Since the LBPMM consists of three phases, three PWM amplifiers Model 12A8K from Advanced Motion Controls are used to power each phase. Three amplifiers are capable of providing the current output $\pm 6 \mathrm{~A}$.

The relation between the provided voltage and the output current is monitored and recorded to determine the gain of each PWM amplifier. This gain is determined by incrementing the input voltage by $1 \mathrm{~V}$, from $10 \mathrm{~V}$ to $-10 \mathrm{~V}$ and recording the current output. Then these data are plotted and fitted into a line. Table 2 shows the result of the determined PWM amplifiers gain.

Table 2

PWM amplifier gains

\begin{tabular}{lccc}
\hline \hline & Phase A & Phase B & Phase C \\
\hline Gain $(\mathrm{A} / \mathrm{V})$ & 0.512 & 0.523 & 0.457 \\
\hline \hline
\end{tabular}

\section{5) Conditioning circuit}

The LVDT generated the output voltage from 0 to $5 \mathrm{~V}$. However, the A/D channel of the dSPACE has the range from $-10 \mathrm{~V}$ to $10 \mathrm{~V}$. To match the voltage range, a circuit was implemented. Also, due to noise considerations, an anti-aliasing filter was implemented to remove some of the noise present. Dr. Kim designed both of these circuits. These two circuits were combined into one circuit. The schematic of the combined circuit is given in Figure 6. 


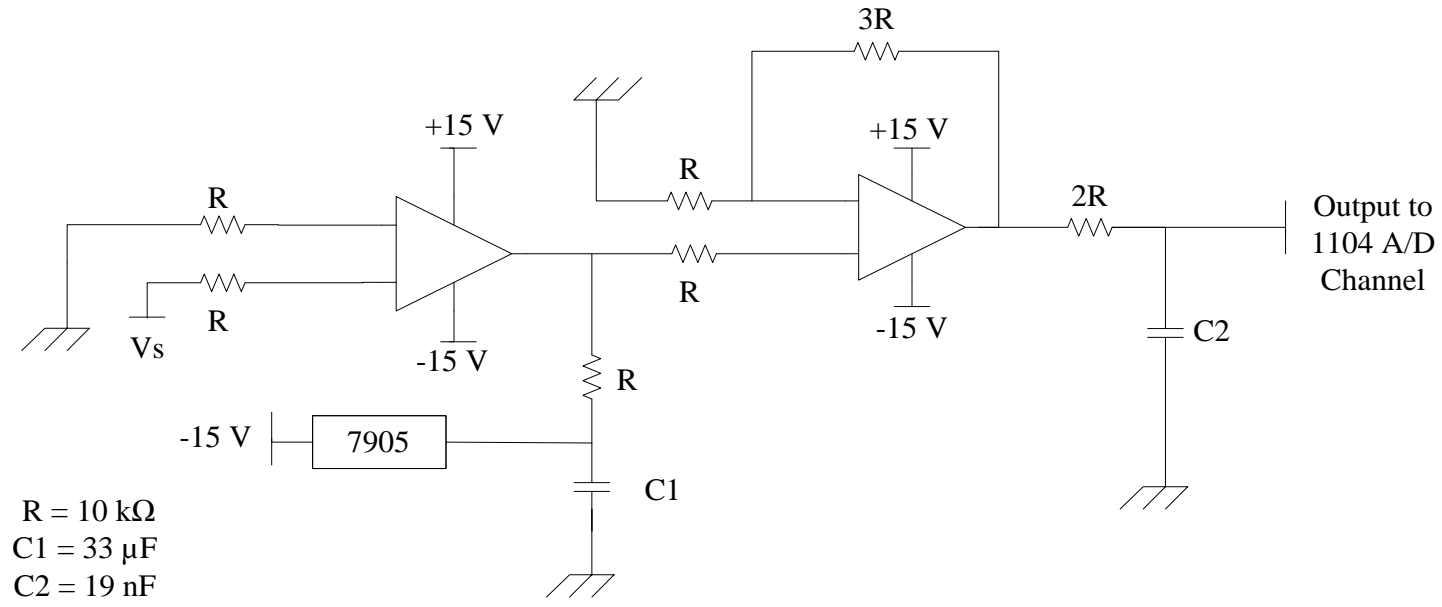

Figure 6. Schematic diagram of the conditioning circuit.

6) dSPACE 1104 control board

The DS1 104 digital-signal-processing (DSP) controller board from dSPACE, Inc. is used for the real-time interfacing between the controller and the quarter-car test bed. The DS1104 board has a 250-MHz Power PC 603e with a Texas Instruments' DSP TMS320F240 chip on it. It contains four 16-bit A/D channels, four 12-bit A/D channels, eight 16-bit D/A channels.

The software used for the actual implementation is Control Desk Developer Version 2.1.1, which connected with Matlab 6.1.0.450 (R12.1) Simulink. This developmental environment supports the graphical user interface (GUI), which allows the data acquisition, process, and monitoring of the sensor signal in real time. 


\section{7) Disturbance generating system}

As in Figure 7, the road disturbance is generated by a rotating cam. This rotating cam induces the vertical movement of the wheel. The end of the cam shaft is connected to the electric drill (Black \& Deker Model\# DR550), which rotates the cam shaft.

Since this electric drill is operated manually, the trigger of this drill should be squeezed with the constant pressure to generate the constant road disturbance. For this, a bolt and a bolt-cast are installed around the drill trigger. As the winding number of the bolt increases, it presses the drill trigger with higher pressure. Accordingly, the cam rotates at a higher frequency. Table 3 shows the relation between the winding of the bolt and the generated disturbance frequencies.

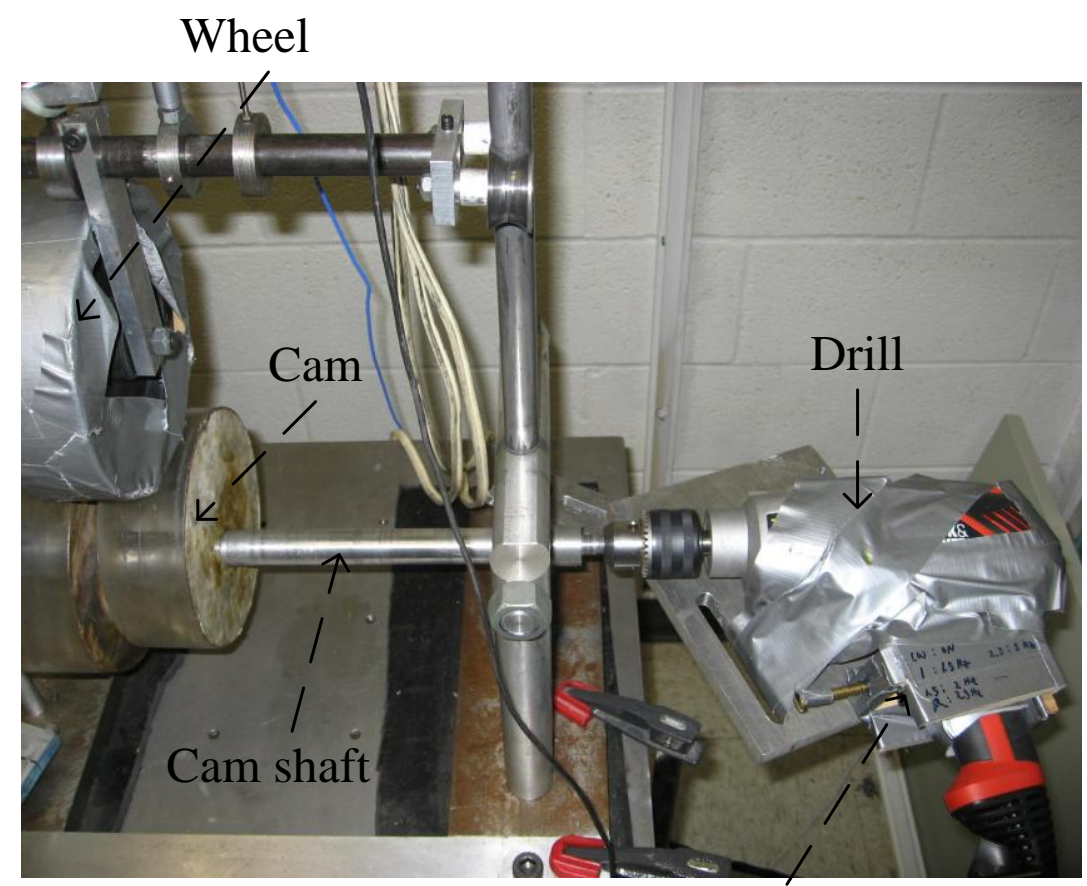

Bolt and bolt cast

Figure 7. Photograph of the disturbance-generating system. 
Table 3

Bolt winding and generated disturbance frequencies

\begin{tabular}{cccc}
\hline \hline & 3 turns & 4.5 turns & 5 turns \\
\hline $\begin{array}{l}\text { Disturbance } \\
\text { frequencies }\end{array}$ & $3.5 \mathrm{~Hz}$ & $4 \mathrm{~Hz}$ & $5 \mathrm{~Hz}$ \\
\hline \hline
\end{tabular}




\section{CONTROL METHODOLOGIES AND EXPERIMENTAL RESULTS}

In this section, four classes of controllers are designed and implemented in the quarter-car test bed, and their experimental results are presented.

\section{A. Modified Lead-Lag Control}

The output of this modified lead-lag controller is three-phase currents. They control the velocity of the sprung mass rather than its position. Since the state-spacebased control sets the velocity of the sprung mass as a reference input for the convenience of the controller design [11], the same reference input is used in all control methodologies for fair comparison of their performances. From (4) and Table 1, the transfer function from $F_{a c t}(t)$ to $\dot{x}_{s}(t)$ is determined as follows.

$$
G(s)=\frac{0.435 s^{3}+9.547 s^{2}+29.79 s+5.831 \times 10^{-14}}{s^{4}+21.95 s^{3}+1398 s^{2}+1.452 \times 10^{4} s+4.531 \times 10^{4}}
$$

The control objectives are as follows. First, a high loop gain is desirable around the operating frequency at $22 \mathrm{rad} / \mathrm{s}$ for good disturbance attenuation and command following. However, this high gain would yield large current flow in the LBPMM, which would raise its temperature and demagnetize the magnets. Therefore, the gain was limited by examining the simulation result of the maximum current flow $( \pm 4 \mathrm{~A})$ in the LBPMM. Finally, the loop gain of the controller at around the operating frequency was determined as $56 \mathrm{~dB}$. 
Second, the control bandwidth was set to be $[10 \mathrm{rad} / \mathrm{s}, 80 \mathrm{rad} / \mathrm{s}]$. Since the openloop frequency response of this quarter car has a low gain in low and high frequencies and a high gain in the middle frequency with two cross-over frequencies, the bandwidth could be adjusted by changing either the lower cross-over frequency or the higher crossover frequency. In this paper, a lag compensation $\left(\frac{0.2252 s+1.15}{s+1.005}\right)$ was applied in the low-frequency range to achieve this goal.

Third, since the gain should be low in the high frequency range to attenuate noise, another lag compensation $\left(\frac{0.04681 s+100.5}{s+100.54}\right)$ was applied. Finally, sufficient gain and phase margins should be obtained due to modeling uncertainties. To achieve this objective, a lead compensation $\left(\frac{1.949 s+100}{s+100.02}\right)$ was introduced between the two lag controllers.

The lower-frequency lag controller yields a lower loop gain. The lead controller around the operating frequency broadens the bandwidth. Therefore, each lead or lag controller should be fine-tuned by examining the overall loop transfer function. To decide the exact corner frequencies in each of the lead or lag controllers, the Matlab SISO (single-input-single-output) tool was used. The modified lead-lag controller with one lead and two lag controllers was finalized in the $s$ domain as (6). Figure 8 shows the frequency response of the loop transfer function. 


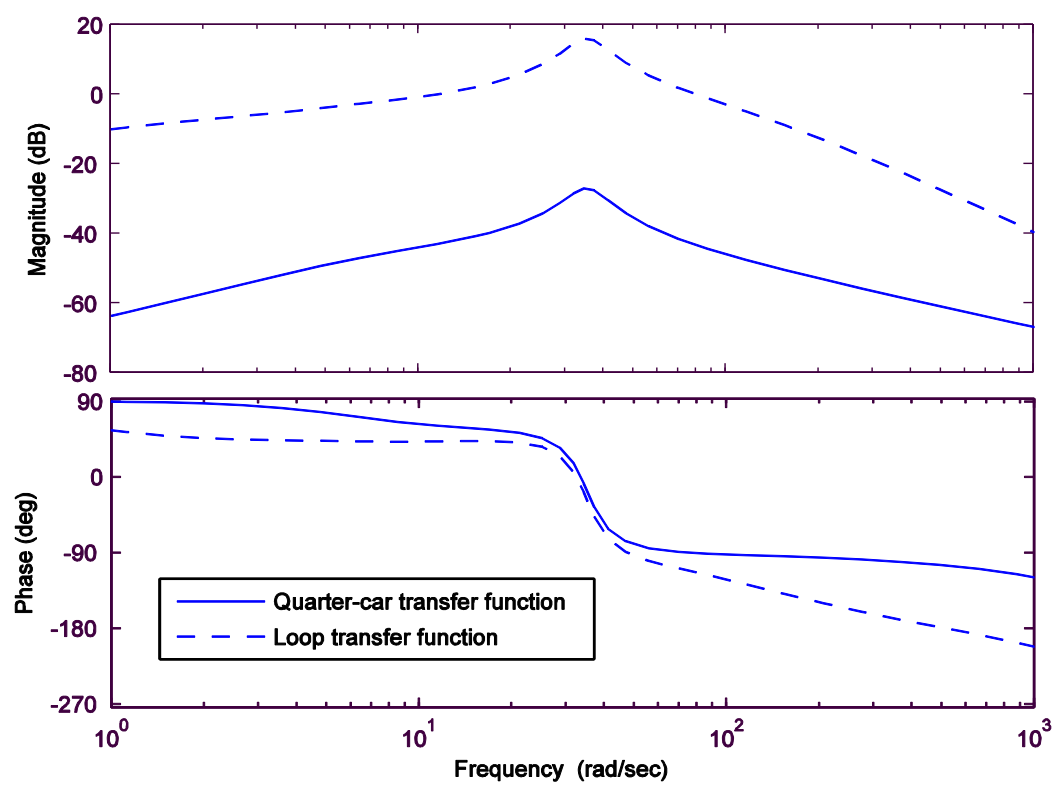

Figure 8. Quarter-car and loop-transfer-function frequency responses of the quarter-car dynamics (5) and the modified lead-lag controller (6). Gain and phase margins are 28.2 $\mathrm{dB}$ and $66.4^{\circ}$, respectively.

$$
C(s)=12 \frac{(s+2147)(s+51.31)(s+5.107)}{(s+100.54)(s+100.02)(s+1.005)}
$$

As seen from Figure 8, the loop-transfer-function gain is higher than that of the open-loop transfer function around the operating frequency $(22 \mathrm{rad} / \mathrm{s})$. The bandwidth is acceptable since it is close to the frequency range of [10 rad/s, $80 \mathrm{rad} / \mathrm{s}]$.

When the quarter-car test bed is under closed-loop control, the LBPMM generates the force to attenuate road disturbance, which results in the current flow in each coil set as shown in Figure 9. The current flow in each coil set is monitored from the Control Desk software. Since the disturbance from the road is a sinusoidal input with a specified frequency, the current flow in the LBPMM would generate the force at the 
same frequency. However, this does not necessarily imply that each current flow in the individual coil set should contain exactly the same and only the frequency component as in the road disturbance due to some unmodeled nonlinear dynamics in the system.

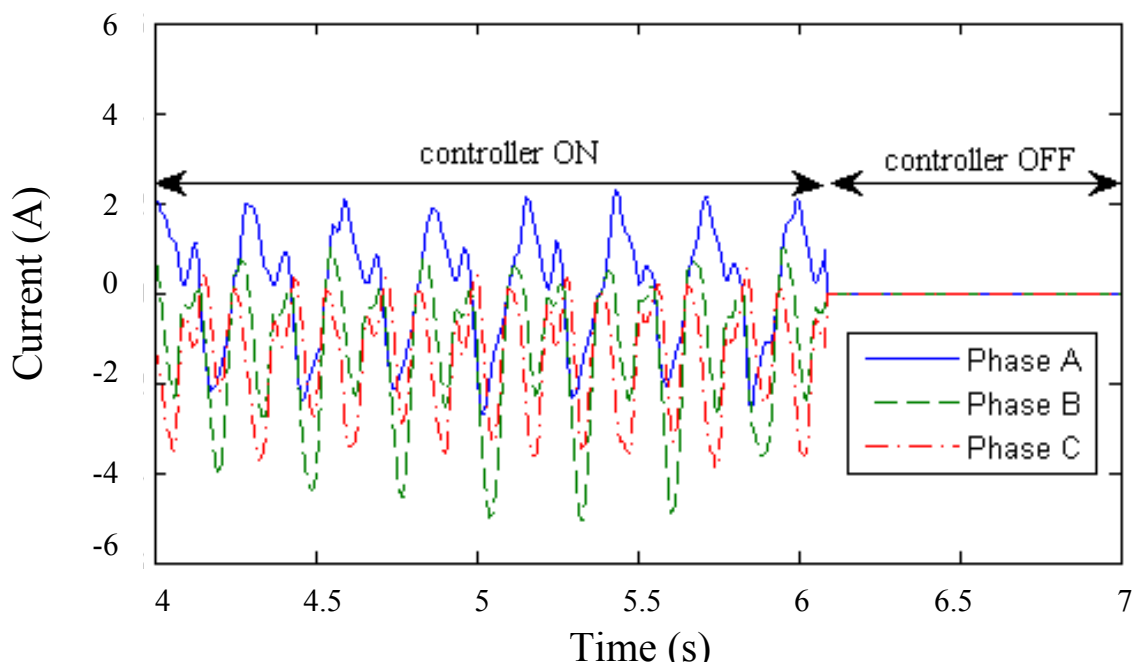

Figure 9. Current flow of the modified lead-lag control in experiment for the $3.5-\mathrm{Hz}$ ( 22 $\mathrm{rad} / \mathrm{s}$ ) disturbance. The LBPMM receives no control input when the controller is turned off.

The simulation and experimental results of disturbance rejection are presented in Figure 10. Due to the modeling uncertainties in the quarter-car model, there are discrepancies between these two results. When the controller is turned off, the road disturbance affects directly to the quarter-car test bed, which results in high-velocity oscillation of the sprung mass. 


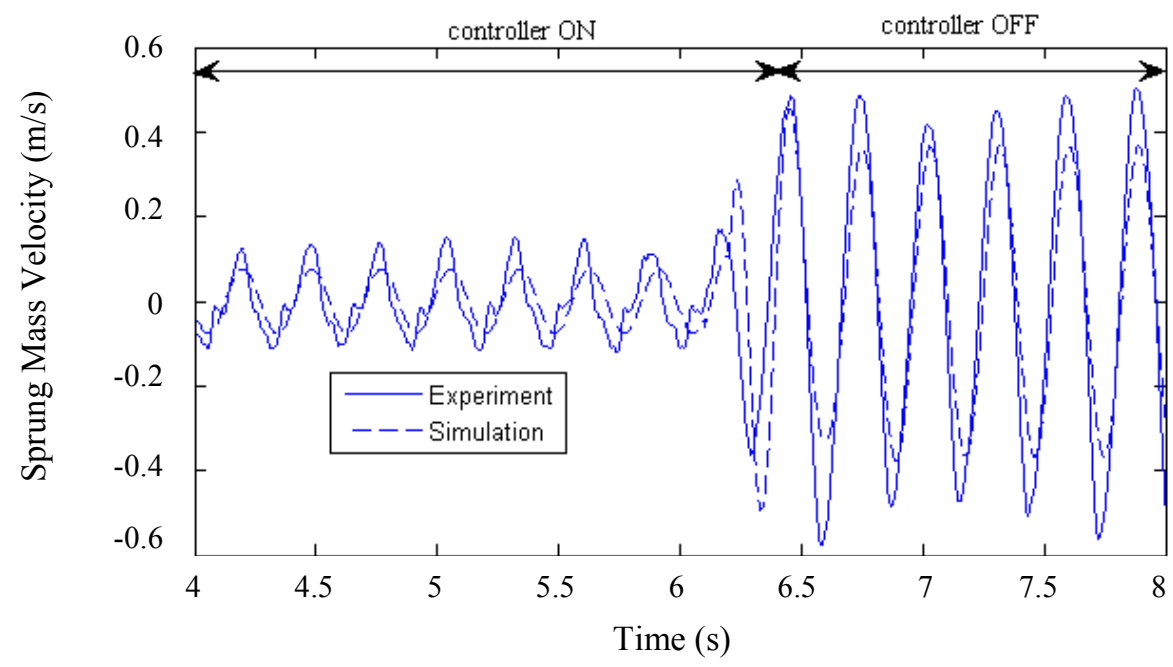

Figure 10. Experiment and simulation results of the modified lead-lag control for the 3.5-Hz disturbance.

\section{B. Linear-Quadratic Servo Control}

LQ servo control is developed by introducing the command input and the output disturbance. From (4), quarter-car model can be expressed as follows.

$$
\begin{aligned}
& \dot{\boldsymbol{x}}_{p}(t)=A_{p} \boldsymbol{x}_{p}(t)+B_{P} u(t) \\
& y_{p}(t)=C_{p} \boldsymbol{x}_{p}(t)
\end{aligned}
$$

where $\boldsymbol{x}_{p}(t)=\left[\dot{x}_{s}(t) \quad \dot{x}_{u s}(t) \quad x_{s}(t)-x_{u s}(t) \quad x_{u s}(t)-x_{r}(t)\right]^{T}$,

$$
A_{p}=\left[\begin{array}{cccc}
0 & 0 & \frac{-k}{M_{s}} & 0 \\
0 & \frac{-c_{w}}{M_{u s}} & \frac{k}{M_{u s}} & \frac{-k_{w}}{M_{u s}} \\
1 & -1 & 0 & 0 \\
0 & 1 & 0 & 0
\end{array}\right], B_{p}=\left[\begin{array}{c}
\frac{1}{M_{s}} \\
\frac{-1}{M_{u s}} \\
0 \\
0
\end{array}\right], C_{p}=\left[\begin{array}{c}
1 \\
0 \\
0 \\
0
\end{array}\right]^{T} \text { as in (4). Thus, } y_{p}(t)=\dot{x}_{s}(t),
$$


and $\boldsymbol{x}_{p}(t)$ is partitioned as follows.

$$
\boldsymbol{x}_{p}(t)=\left[y_{p}(t) \mid \boldsymbol{x}_{\boldsymbol{R}}^{T}(t)\right]^{T}=\left[\dot{x}_{s}(t) \mid \dot{x}_{u s}(t) \quad x_{s}(t)-x_{u s}(t) \quad x_{u s}(t)-x_{r}(t)\right]^{T}
$$

The vertical line indicates that $y_{p}(t)=\dot{x}_{s}(t)$.

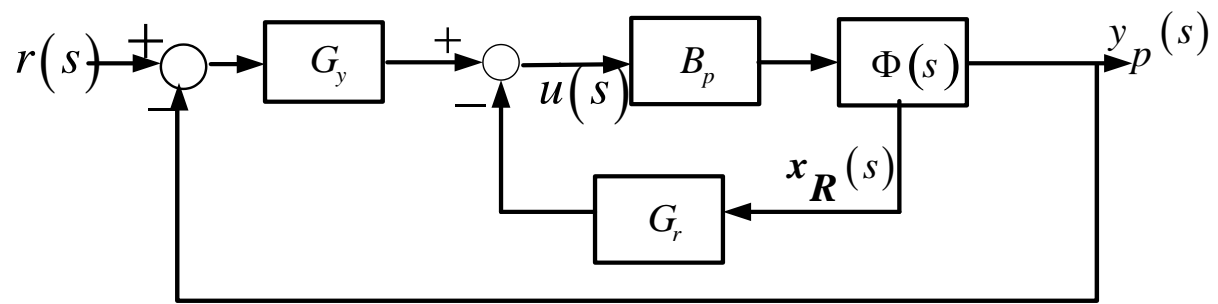

(a)

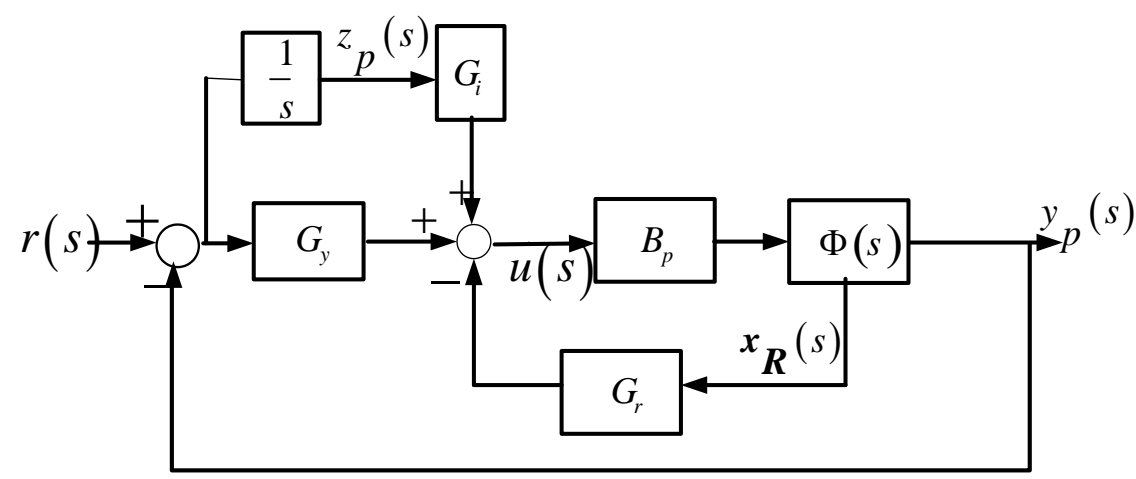

(b)

Figure 11. Block diagrams of (a) the standard LQ servo control and (b) the LQ servo control with an integrator.

As shown in Figure 11, the control gain matrices $G_{y}$ and $G_{r}$ are applied to $y_{p}(s)$ and $\boldsymbol{x}_{\boldsymbol{R}}(s)$, respectively. To eliminate a non-zero steady-state error for the step command input or output disturbance, this LQ servo controller is implemented with an integrator as shown in Figure 11 (b). In this application, the LQ servo model is 
determined by considering the frequency responses of the loop transfer functions of these two models given in Figure 12.

As shown in Figure 11 (b), the control gains for the integrator, output state, and rest states are $G_{i}, G_{y}, G_{r}$, respectively [22]. This LQ servo system consists of the standard LQ servo dynamics (7) and the integrator dynamics. With $r(s)=0$ in a regulation problem,

$$
z_{p}(s)=-\frac{I}{s} y_{p}(s)=-\frac{I}{s} \dot{x}_{s}(s)
$$

The augmented system is defined as follows.

$$
\dot{x}(t)=A x(t)+B u(t)
$$

where $\boldsymbol{x}(t)=\left[\begin{array}{lll}z_{p}(t) & y_{p}(t) \boldsymbol{x}_{R}{ }^{T}(t)\end{array}\right]^{T}, A=\left[\begin{array}{cc}0 & -C_{P} \\ 0 & A_{P}\end{array}\right]$ and $B=\left[\begin{array}{ll}0 & B_{p}{ }^{T}\end{array}\right]^{T}$. The control law is defined as follows.

$$
u(t)=-G x(t)
$$

where $G=\left[\begin{array}{lll}G_{i} & G_{y} & G_{r}\end{array}\right]$.

To obtain $G$, a control algebric Riccati equation (CARE) should be solved. To construct this CARE, a symmetric positive definite matrix $R$ and a symmetric positive semi-definite matrix $Q$ should be determined. The $R$ matrix affects the loop gain that determines the system bandwidth. Although a large $R$ is desirable in terms of command following and disturbance rejection, it results in a large control input that yields higher 
power consumption in the LBPMM. The maximum current flow is constrained at $\pm 4 \mathrm{~A}$ the same as the case of the modified lead-lag controller. After several design iterations, $R$ was set to be 0.005 . The diagonal elements of the $Q$ matrix are the weights of each state and determine the shape of the loop transfer function. Since the second state $\left(\dot{x}_{s}(t)\right)$ should be regulated, the $Q$ matrix is desirable to have a larger element $Q(2,2)$ than other elements in the $Q$ matrix. After several design iterations, the $Q$ matrix was determined as follows.

$$
\begin{aligned}
& \operatorname{diag}(Q)=\left[\begin{array}{lllll}
0.01 & 170 & 0.01 & 0.01 & 0.01
\end{array}\right] \\
& Q_{i j}=0 \text { for } i \neq j
\end{aligned}
$$

A unique positive semi-definite symmetric matrix $K$ is determined by the following CARE.

$$
-K A-A^{T} K-Q+K B R^{-1} B^{T} K=0
$$

$K$ is solved with Matlab as follows.

$$
K=\left[\begin{array}{ccccc}
1.1078 & 0 & 0 & -1.1078 & -1.1078 \\
0 & 0 & 0 & -0.0003 & 0 \\
0 & 0 & 0 & -0.0003 & 0 \\
-1.1078 & -0.0003 & -0.0003 & 1.1190 & 1.1104 \\
-1.1078 & 0 & 0 & 1.1104 & 1.1102
\end{array}\right]
$$

The feedback gain $G$ is determined as follows.

$$
G=R^{-1} B K=\left[\begin{array}{lllll}
0.0013 & 147.58 & -25.7212 & 0 & 272.5471
\end{array}\right]
$$


Figure 12 shows the loop transfer functions of a standard LQ servo model (i.e. model $\underline{a}$ ) and an LQ servo model with an integrator (i.e. model $\underline{b}$ ) with the feedback gain from (15). The most significant difference between these two models is the lowfrequency response. Model $\underline{b}$ has the slope of the magnitude plot of $20 \mathrm{~dB} /$ decade around the lower cross-over frequency. Model $\underline{a}$ has a larger slope than $20 \mathrm{~dB} /$ decade around the lower cross-over frequency. Therefore, the magnitude of the sensitivity function of the model $\underline{a}$ is smaller than model $\underline{b}$. Model $\underline{a}$ is desirable in terms of disturbance rejection and command following. However, improvement of the sensitivity in some range deteriorates the sensitivity in another frequency range. The system could also become unstable due to this deterioration [20]. Since the operating frequency of the quarter-car model is around $22 \mathrm{rad} / \mathrm{s}$, improvement of the sensitivity in the frequency range less than $22 \mathrm{rad} / \mathrm{s}$ is not as significant factor as the stability of the system. Therefore, model $\underline{b}$ is more suitable for the quarter-car than model $\underline{a}$.

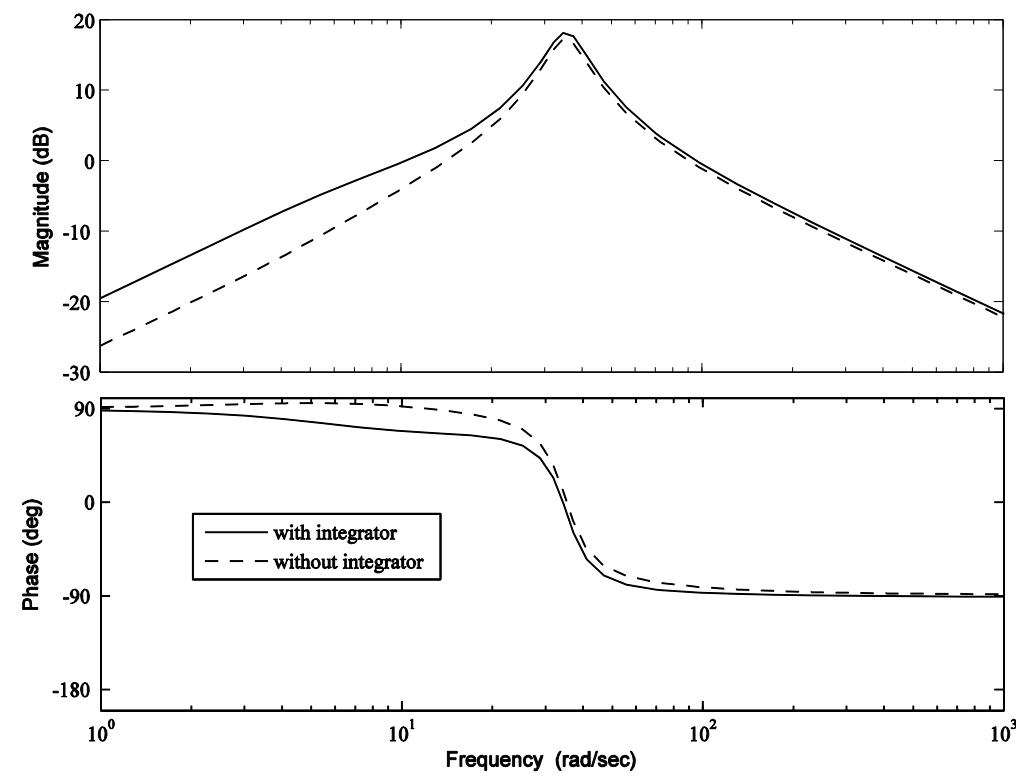

Figure 12. Frequency responses of the loop transfer functions in the LQ servo control. 
Its control objectives are similar to those of the modified lead-lag control. First, the loop gains should be high around the operating frequency. Second, the control bandwidth should be located in $[10 \mathrm{rad} / \mathrm{s}, 80 \mathrm{rad} / \mathrm{s}]$. The control objectives are more conveniently achievable with model $\underline{b}$ than model $\underline{a}$ because it has an additional design parameter $\left(G_{i}\right)$. This also gives the relevance to the usage of the integrator.

\section{1) Kalman filter design}

LQ servo requires full state feedback. The last state of the system is defined as the tire deflection $\left(x_{u s}(t)-x_{r}(t)\right)$. This state is difficult to be measured because the magnitude is small and locating the sensor is not convenient. Therefore, it is estimated by a Kalman filter. This estimator requires the measured output $\left(\dot{x}_{s}(t)\right)$ and the system

control input $F_{a c t}(t)$ as an estimator input. To solve the filter algebric Riccati equation (FARE) and obtain the Kalman-filter gain, a positive value $\Theta$ and a non-negative value $\Xi$ should be determined in (17). With initial values of $\Theta=1$ and $\Xi=L^{T} L$, they were adjusted and determined as $\Theta=0.00001$ and $\Xi=0.01$ after several design iterations. As expressed in (4), the output disturbance affects the last state of the quarter-car model. Therefore, the matrix $L$ is defined as follows.

$$
L=\left[\begin{array}{llll}
0 & 0 & 0 & -1
\end{array}\right]^{T}
$$

Then the unique positive semi-definite symmetric matrix $P$ is determined by solving the following FARE. 


$$
A P+P A^{T}+L \Xi L^{T}-P C^{T} \Theta^{-1} C P=0
$$

The solution is obtained as follows by Matlab 'CARE' function.

$$
P=\left[\begin{array}{cccc}
0.0005 & 0.0004 & 0 & -0.0003 \\
0.0004 & 0.0012 & 0 & -0.0008 \\
0 & 0 & 0 & 0 \\
-0.0003 & -0.0008 & 0 & 0.0007
\end{array}\right]
$$

The Kalman-filter gain $H$ is determined as follow.

$$
H=P C^{T} \Theta^{-1}=\left[\begin{array}{llll}
46.9286 & 41.9643 & -1.6644 & -28.9742
\end{array}\right]
$$

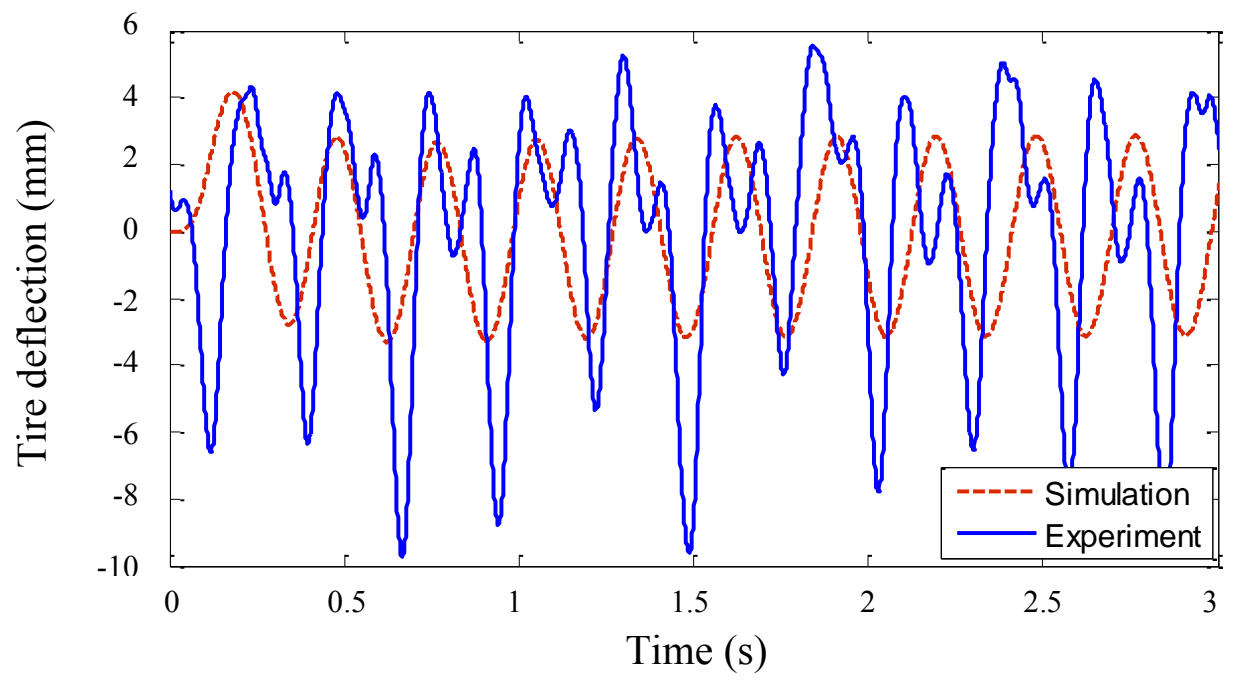

Figure 13. Estimated state comparison between simulation and experiment results.

Figure 13 shows the estimated tire deflection $\left(x_{u s}(t)-x_{r}(t)\right)$ by the Kalman-filter algorithm in closed-loop control. The solid line represents the data generated from the Control Desk during the experiment. The dashed line represents the data generated from the Simulink block without experiment. There is some discrepancy between the 
simulation and experimental results of state estimation. In the Kalman-filter algorithm, the measured output and the disturbance are assumed as zero-mean white Gaussian noises. In the quarter-car model, there is some discrepancy between the measured output $\left(\dot{x}_{s}(t)\right)$ and the zero-mean white Gaussian noise (Figures 10 and 14), which limits the performance of the state estimator. Also, noises from the sensors are the other reason for this discrepancy. Since the LQ servo controller requires more sensors (two accelerometers and one LVDT) than the other controllers (one accelerometer) and data from each sensor contains the noise, LQ servo controller affected by the sensor noise. The performance of the disturbance attenuation is presented in Figure 14. Due to the error from the state estimator, disturbance attenuation contains some discrepancy between the experiment and simulation results.

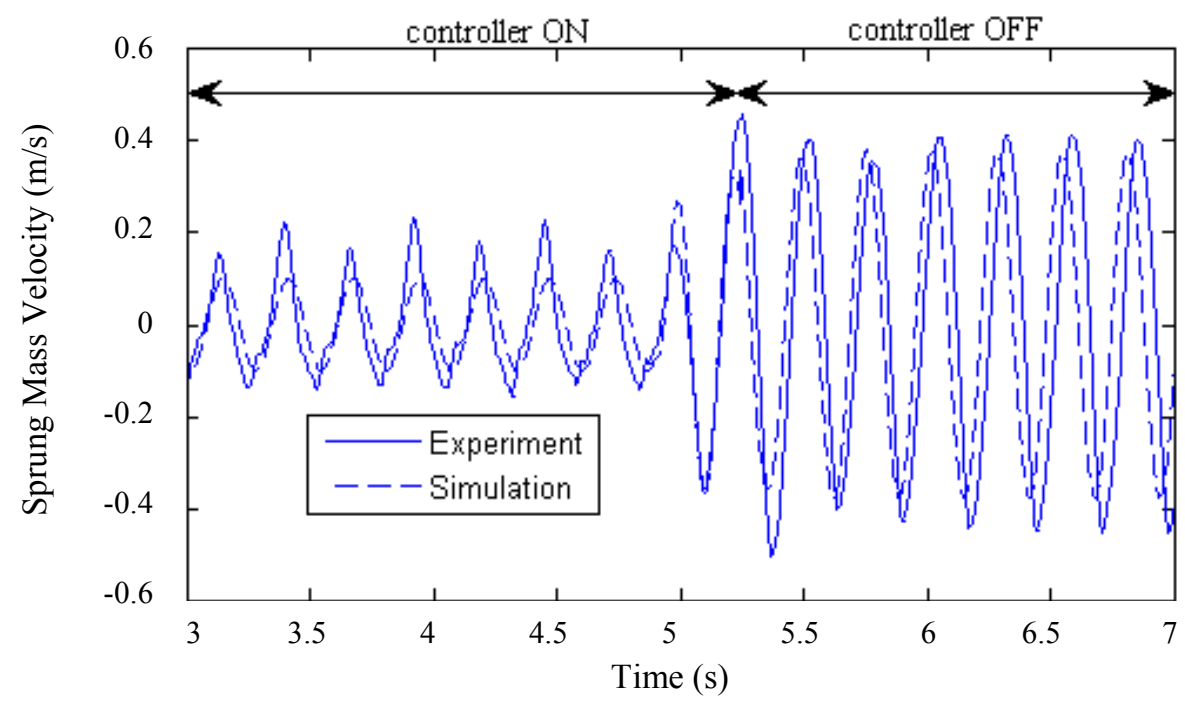

Figure 14. Experiment and simulation results of the LQ servo control. 


\section{Fuzzy Control}

A Mamdani-type fuzzy controller is implemented in this section [23]. The input to this fuzzy controller is the system error $(e(t))$, and the output is the control input

$\left(F_{a c t}(t)\right)$. To determine $F_{a c t}(t), e(t)$ is fuzzified by the membership functions shown in Figure 15 (a) and defuzzified by the membership functions shown in Figure 15 (b). The membership functions for the fuzzification are denoted according to the amount of the error: NLE (Negative Large Error), NME (Negative Medium Error), NSE (Negative Small Error), ESE (Evenly Small Error), PSE (Positive Small Error), PME (Positive Medium Error), and PLE (Positive Large Error). For defuzzification, membership functions are denoted according to the force generated by each membership function: NLF (Negative Large Force), NMF (Negative Medium Force), NSF (Negative Small Force), ESF (Evenly Small Force), PSF (Positive Small Force), PMF (Positive Medium Force), and PLF (Positive Large Force). The area under the membership functions (NLF, NMF, NSF, ESF, PSF, PMF, PLF $)$ are defined by $\mu_{i}(i=1,2, \ldots, 7)$. The area under the membership functions are required to calculate the generated force by the center of gravity (COG) method.

The domains for the fuzzification and the defuzzification are determined from the previously obtained results. The range of error in Figure 15 (a) was set as $[-0.8,0.8]$ because the magnitude of the largest measured error $\left(\left|\dot{x}_{s}(t)\right|\right)$ was less than $0.8 \mathrm{~m} / \mathrm{s}$. The range of outputs in Figure 15 (b) was set as $[-30,30]$ because the LBPMM could generate force up to near $\pm 30 \mathrm{~N}$. 


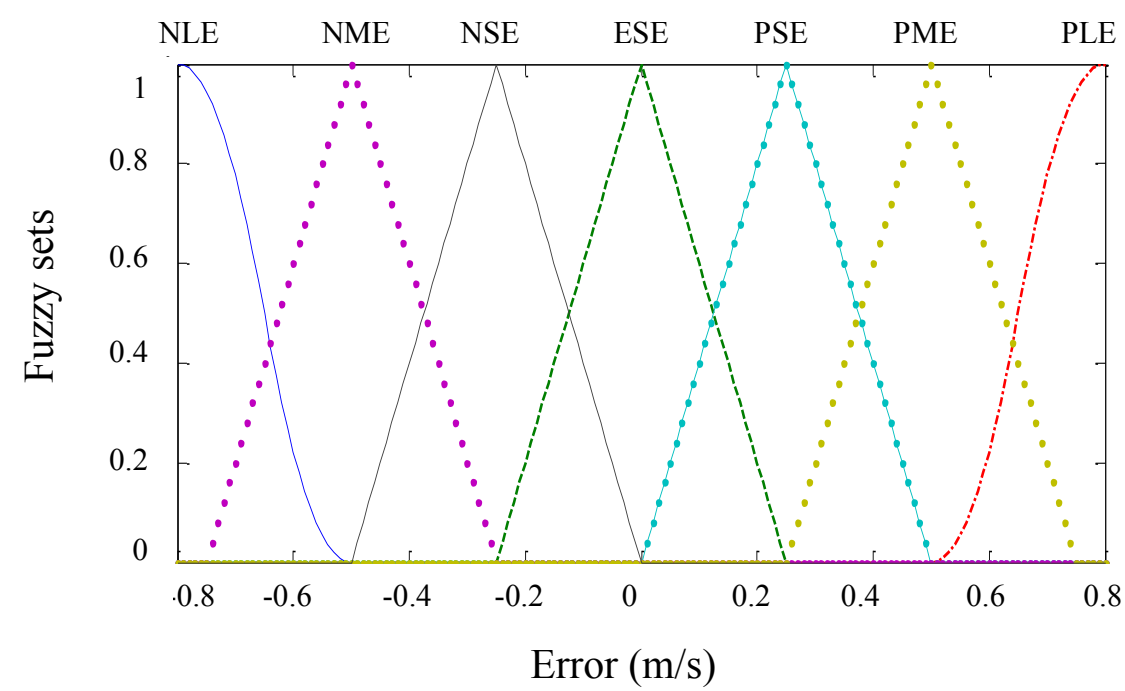

(a)

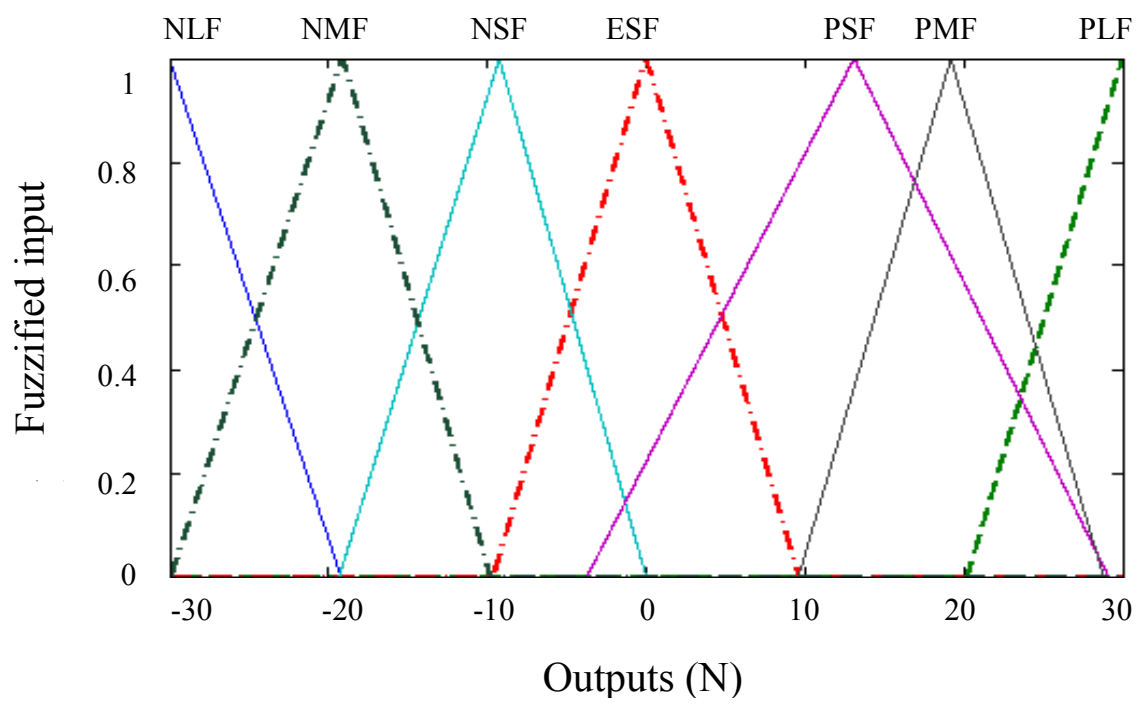

(b)

Figure 15. (a) Membership functions for fuzzification. (b) Membership functions for defuzzification.

As presented in Figure 15, seven membership functions were implemented for the fuzzification and defizzification. Several controllers with the different numbers of 
membership functions were tested, and the one with seven membership functions was selected since it showed the smallest sprung mass velocity under the closed-loop control.

Table 4

Rules of the fuzzy controller

\begin{tabular}{l|lllllll}
\hline \multicolumn{1}{c|}{ Error } & \multirow{2}{*}{ NLE } & NME & NSE & ESE & PSE & PME & PLE \\
\hline NLF & 1 & 0 & 0 & 0 & 0 & 0 & 0 \\
NMF & 0 & 1 & 0 & 0 & 0 & 0 & 0 \\
NSF & 0 & 0 & 1 & 0 & 0 & 0 & 0 \\
ESF & 0 & 0 & 0 & 1 & 0 & 0 & 0 \\
PSF & 0 & 0 & 0 & 0 & 1 & 0 & 0 \\
PMF & 0 & 0 & 0 & 0 & 0 & 1 & 0 \\
PLF & 0 & 0 & 0 & 0 & 0 & 0 & 1 \\
\hline \hline
\end{tabular}

Table 4 shows the rules of this fuzzy controller. Since this active-suspension test bed is a single-input, single-output system, the input and the output forms single-dimension arrays. Each fuzzified value is one-to-one matched for the defuzzification. For example, if the error is NLE, the output is NLF. Each rule has the same weight.

The control input as the result of this fuzzy controller is determined by the COG method. The COG method computes $F_{a c t}(t)$ as follows [23].

$$
F_{a c t}(t)=\sum_{i=1}^{7} g_{i} \int \mu_{i} / \sum_{i=1}^{7} \int \mu_{i}
$$

where $g_{i}$ is defined as the COG of the each membership function.

There are non-ideal conditions which yields the unexpected behavior of the quarter-car test bed. For example, vertical and horizontal supporters (Figure 2) are not 
perfectly vertical and horizontal. Since the movement of the sprung mass is guided by those supporters, the movement of the sprung mass also contains non-ideal aspect. Besides of the non-ideality from the supporters, other non-ideal conditions are also arisen from the machining process. The combined non-ideal conditions induced the phenomenon expressed as follows.

When the active-suspension system is under closed-loop control, the sprung mass's maximum absolute velocity is larger when its velocity is positive compared to that with a negative velocity. This phenomenon was observed in both the modified leadlag control and the LQ servo control (Figures 10 and 14). This phenomenon indicates that additional control input is required to attenuate disturbance when the velocity of the sprung mass is positive.

To solve the problem this phenomenon, a membership function PSF in Figure 15 (b) was widened. The PSF is the most significant membership function with the system under closed-loop control because the domain of the PSE covers a small positive error and the PSF is determined by the PSE. The widened PSF induces the increased area of the PSF $\left(\mu_{3}\right)$. Consequently, the absolute value of the COG of the PSF increased. Finally, the magnitude of $F_{a c t}(t)$ also increased by $(20)$ when $0.1<$ Error $(\mathrm{m} / \mathrm{s})<0.4$. 


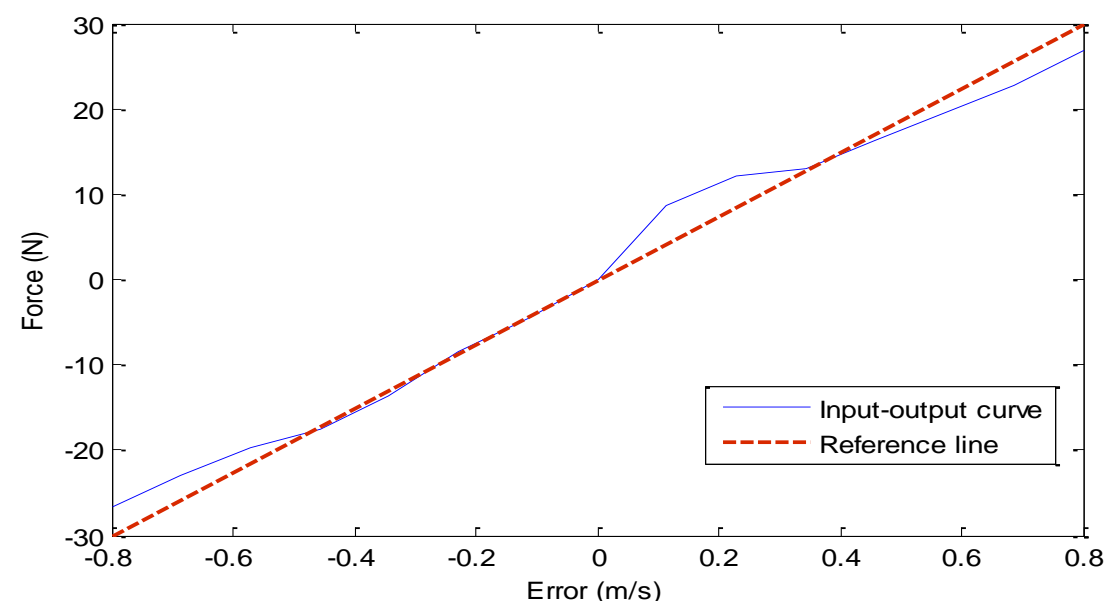

Figure 16. Input-output relation of the asymmetric fuzzy controller.

Figure 16 shows the relation between the error (input) and the generated control force (output). This input-output curve was designed not to be symmetric with respect to the origin. In Figure 17, the previously mentioned phenomenon is reduced in comparison with Figures 10 and 14 due to the additional control input generated in the hump $0.1<$ Error $(\mathrm{m} / \mathrm{s})<0.4$ in Figure 16.

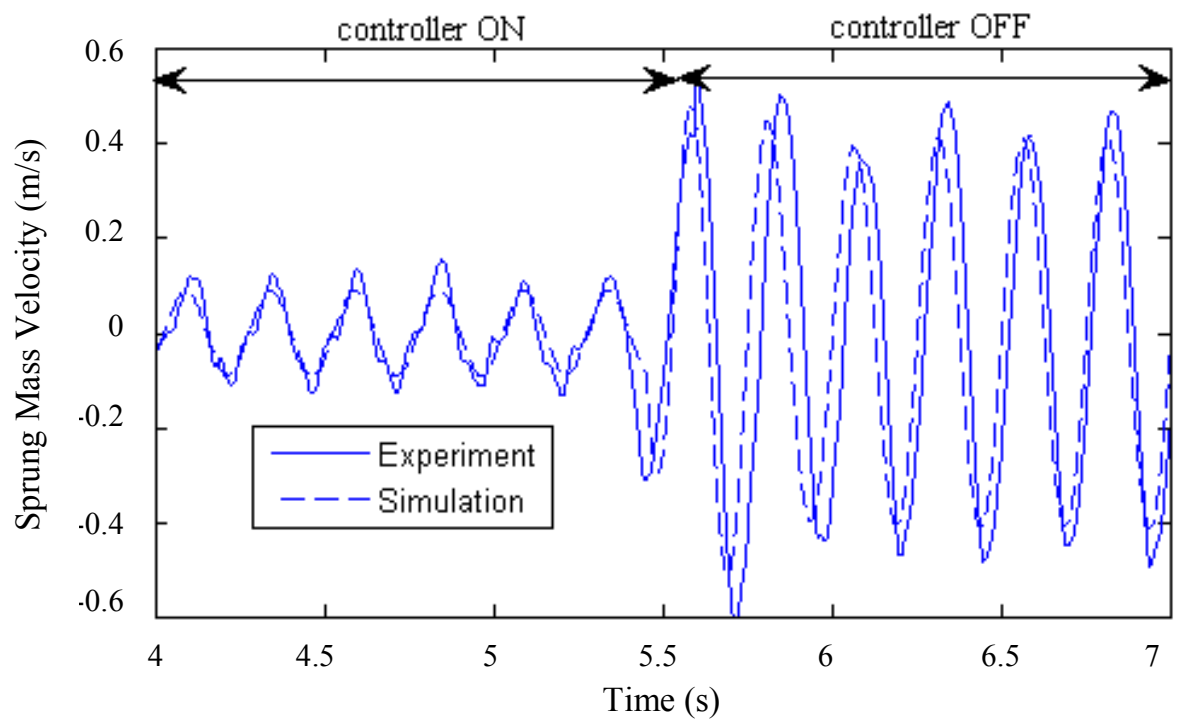

Figure 17. Experiment and simulation results of the fuzzy control. 
Figure 18 and Figure 19 visualize the effect of the asymmetric membership functions when the errors are $-0.1 \mathrm{~m} / \mathrm{s}$ and $0.1 \mathrm{~m} / \mathrm{s}$, respectively. When error is $-0.1 \mathrm{~m} / \mathrm{s}$, only NSE (fourth row in (a)) and NME (fifth row in (a)) have the value in Figure 18. Since NSE matched to NSF (fourth row in (b)) and NME matched to NMF (fifth row in (b)), NSF and NMF are selected for the defuzzification. According to the defuzzification scheme expressed (20), the areas under the NSF and NMF are calculated. Finally, COG of the calculated area is obtained. In this case, generated force is $-4.04 \mathrm{~N}$.

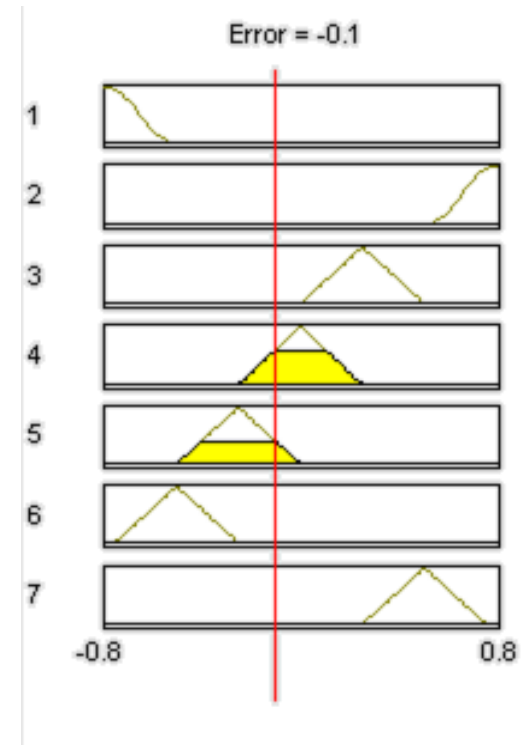

(a)

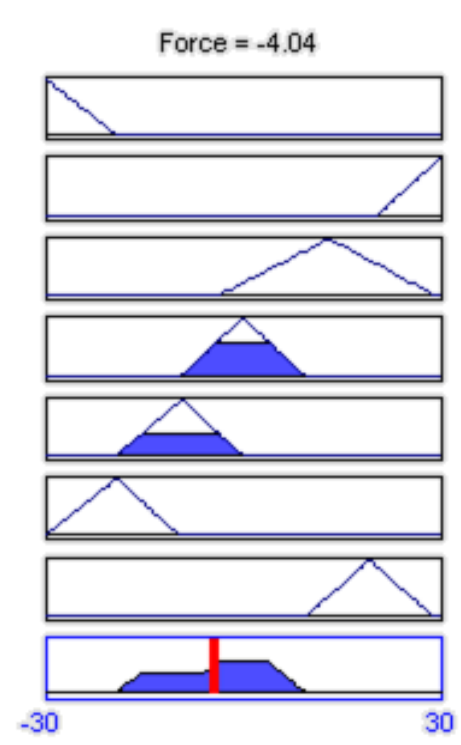

(b)

Figure 18. Generated force from the asymmetric membership functions for $-0.1 \mathrm{~m} / \mathrm{s}$ error. The column (a) and (b) shows the membership functions for the fuzzyfication and the defuzzification, respectively. 


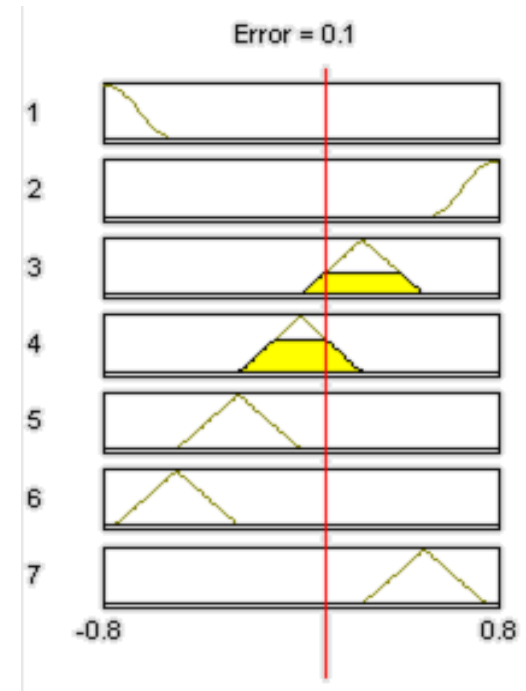

(a)

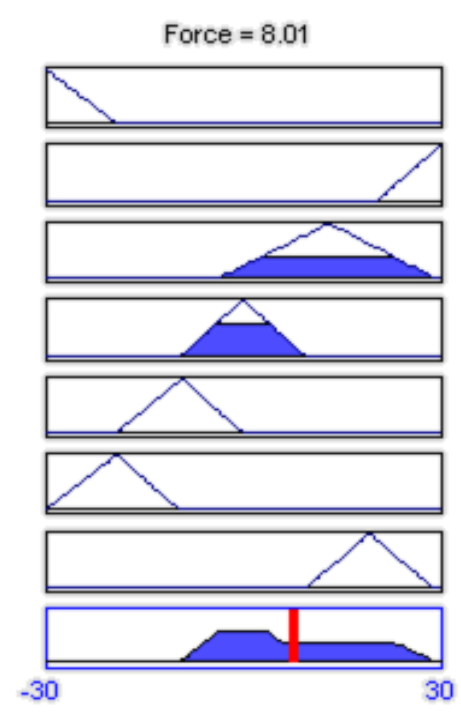

(b)

Figure 19. Generated force from the asymmetric membership functions for $0.1 \mathrm{~m} / \mathrm{s}$ error. The column (a) and (b) shows the membership functions for the fuzzyfication and the defuzzification, respectively.

The generated force for the $0.1 \mathrm{~m} / \mathrm{s}$ error is calculated from the Figure 19 with a similar process. In this case, generated force is $8.01 \mathrm{~N}$ rather than $-4.04 \mathrm{~N}$. It is due to the extended PSF (third row in (b)).

\section{Sliding-Mode Control}

A SMC is developed in this section. The fundamental difference of this controller from the other controllers is that this controller regulates the position of the sprung mass while the other controllers regulate the velocity of the sprung mass. It might be possible for the SMC to control the velocity of the sprung mass also. However, the position of the sprung mass was chosen to be regulated since the construction of the switching function 
is more convenient when the position of the sprung mass is regulated rather than the velocity of the sprung mass.

Without the disturbance input, (4) is presented as follows.

$$
\left[\begin{array}{c}
\ddot{x}_{s}(t) \\
\ddot{x}_{u s}(t) \\
\dot{x}_{s}(t)-\dot{x}_{u s}(t) \\
\dot{x}_{u s}(t)-\dot{x}_{r}(t)
\end{array}\right]=\left[\begin{array}{cccc}
0 & 0 & \frac{-k}{M_{s}} & 0 \\
0 & \frac{-c_{w}}{M_{u s}} & \frac{k}{M_{u s}} & \frac{-k_{w}}{M_{u s}} \\
1 & -1 & 0 & 0 \\
0 & 1 & 0 & 0
\end{array}\right]\left[\begin{array}{c}
\dot{x}_{s}(t) \\
\dot{x}_{u s}(t) \\
x_{s}(t)-x_{u s}(t) \\
x_{u s}(t)-x_{r}(t)
\end{array}\right]+\left[\begin{array}{c}
\frac{1}{M_{s}} \\
\frac{-1}{M_{u s}} \\
0 \\
0
\end{array}\right] F_{a c t}(t)
$$

Equation (21) can be converted to the following form.

$$
\left[\begin{array}{c}
\ddot{x}_{s}(t) \\
\ddot{x}_{u s}(t)
\end{array}\right]=\left[\begin{array}{c}
\frac{-k}{M_{s}}\left(x_{s}(t)-x_{u s}(t)\right) \\
\frac{k}{M_{u s}} x_{s}(t)-\frac{k-k_{w}}{M_{u s}} x_{u s}(t)-\frac{c_{w}}{M_{u s}} \dot{x}_{u s}(t)
\end{array}\right]+\left[\begin{array}{c}
\frac{1}{M_{s}} \\
-\frac{1}{M_{u s}}
\end{array}\right] F_{a c t}(t)
$$

Set the output as $x_{s}(t)$, and (22) could be expressed as follow.

$$
\begin{gathered}
\ddot{X}=F(x, \dot{x})+B u, \\
y=C X \\
\text { where } X=\left[\begin{array}{c}
x_{s}(t) \\
x_{u s}(t)
\end{array}\right], F=\left[\begin{array}{c}
\frac{-k}{M_{s}}\left(x_{s}(t)-x_{u s}(t)\right) \\
\frac{k}{M_{u s}} x_{s}(t)-\frac{k-k_{w}}{M_{u s}} x_{u s}(t)-\frac{c_{w}}{M_{u s}} \dot{x}_{u s}(t)
\end{array}\right], B=\left[\begin{array}{c}
\frac{1}{M_{s}} \\
1 \\
-C=[10], u=F_{a c t}(t) .
\end{array}\right]
\end{gathered}
$$

Assuming that reference input is zero, a switching function and its derivative are defined as follow. The following switching function $s$ is used to decide which control law is to be used at every point in the phase plane. 


$$
\begin{aligned}
& s=w_{1} y+w_{2} \dot{y} \\
& \dot{s}=w_{1} \dot{y}+w_{2} \ddot{y}=w_{1} \dot{y}+w_{2} C F+w_{2} C B u,
\end{aligned}
$$

where $w_{1}$, and $w_{2}$ are assumed to be positive controller gains. Among infinitely many candidates, a Lyapunov function is chosen as follows since it requires a small computational load.

$$
V=\frac{1}{2} s^{2}
$$

Take time derivative of the Lyapunov function results in

$$
\dot{V}=s \dot{s}=s\left(w_{1} \dot{y}+w_{2} C F+w_{2} C B u\right)
$$

From the Lyapunov stability criteria, $\dot{s}$ should be negative for the positive $s$ and vice versa. To make the system always stable, i.e. $\dot{V}<0$, This requirements are expressed as follows.

$$
\begin{gathered}
w_{1} \dot{y}+w_{2} C F+w_{2} C B u<0 \text {, i.e. } u<\left[w_{2} C B\right]^{-1}\left(-w_{2} C F-w_{1} \dot{y}\right) \text { when } s>0 \\
w_{1} \dot{y}+w_{2} C F+w_{2} C B u \geq 0, \text { i.e. } u \geq\left[w_{2} C B\right]^{-1}\left(-w_{2} C F-w_{1} \dot{y}\right) \text { when } s \leq 0
\end{gathered}
$$

To satisfy the requirements (27) and (28), a signum function $\alpha \operatorname{sgn}(s)$ is introduced where $\alpha$ is a positive number. With (27), (28), and this signum function, a variable structure of the control law is expressed as follows.

$$
u=\left[w_{2} C B\right]^{-1}\left\{-w_{2} C F-w_{1} \dot{y}-\alpha \operatorname{sgn}(s)\right\},
$$

where $\operatorname{sgn}(s)=\left\{\begin{aligned} 1 & \text { if } s>\varepsilon \\ -1 & \text { if } s<-\varepsilon\end{aligned}\right.$. 
With an appropriate $\alpha$, equation (29) satisfy the conditions (27), (28) simultaneously under the varying switching function.

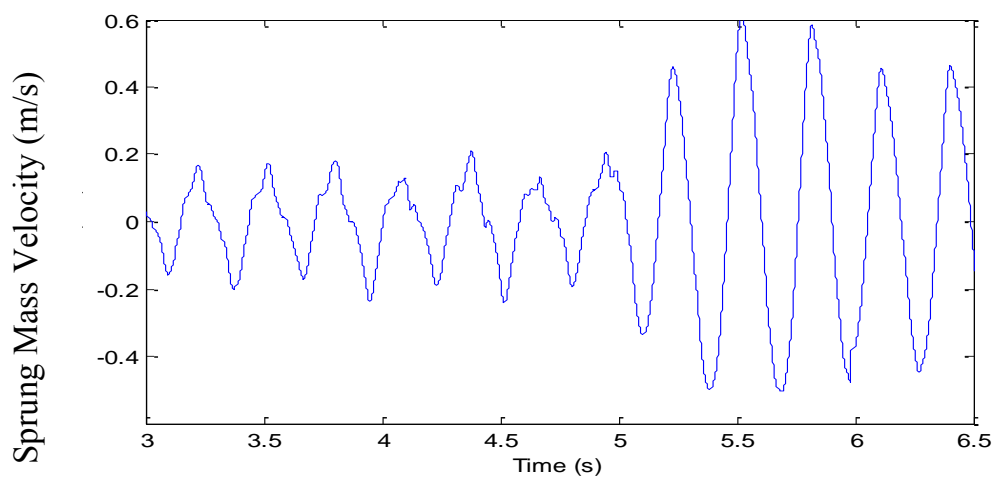

(a)

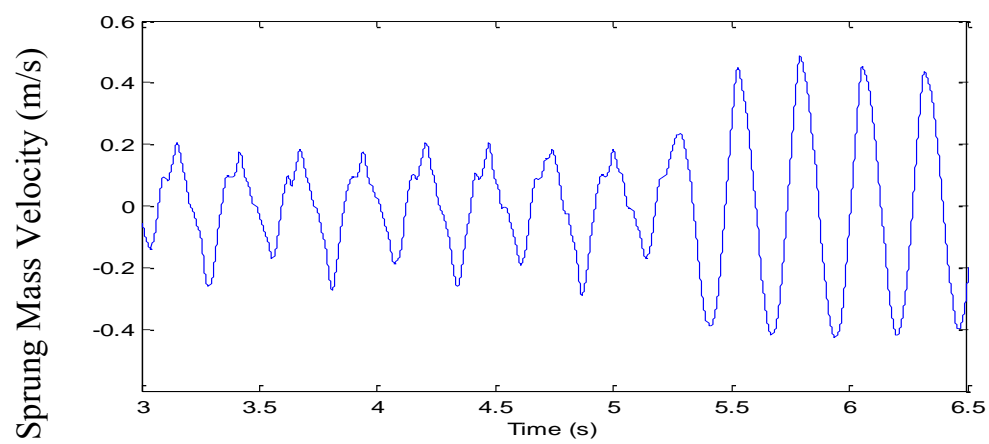

(b)

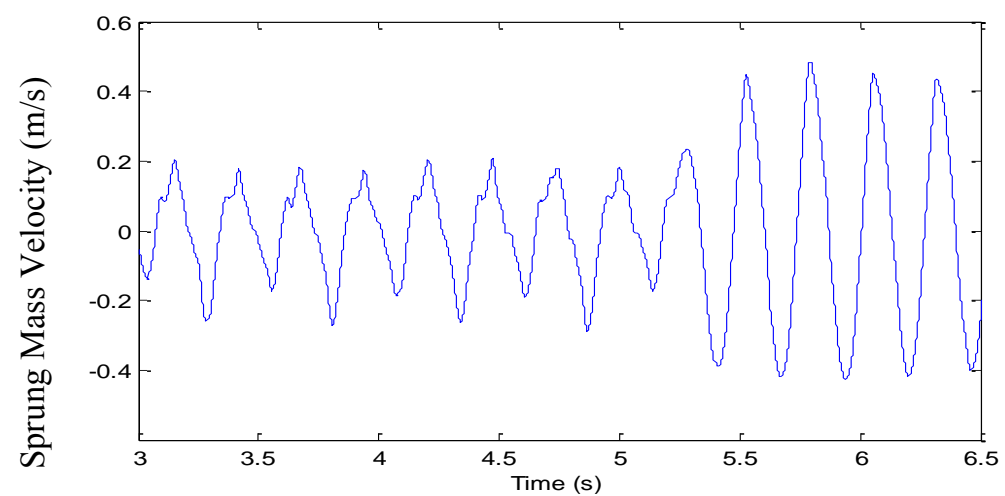

(c)

Figure 20. SMC result in case of (a) $\varepsilon=0.002$, (b) $\varepsilon=0.004$, and (c) $\varepsilon=0.006$, respectively. 


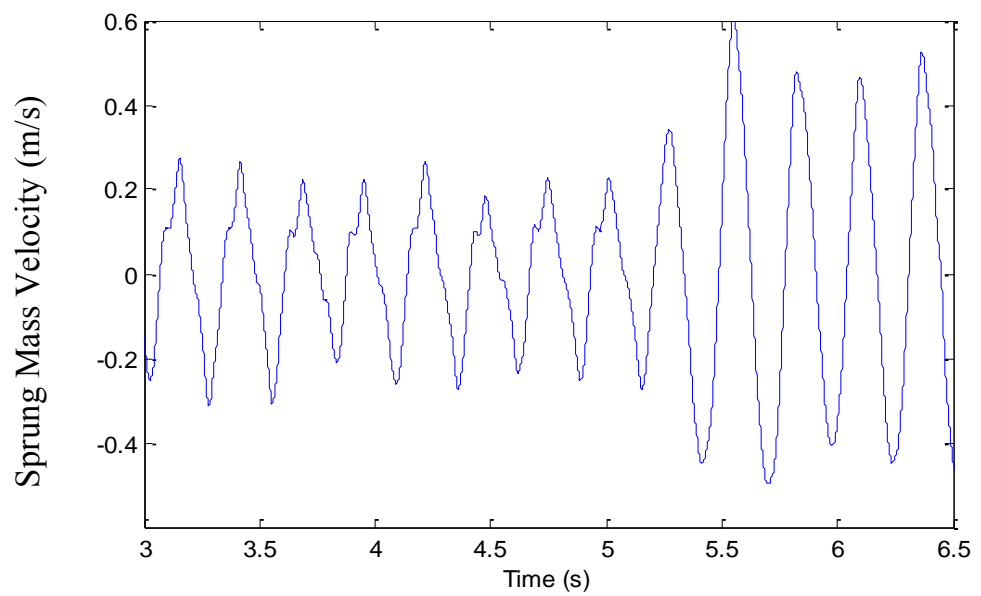

(a)

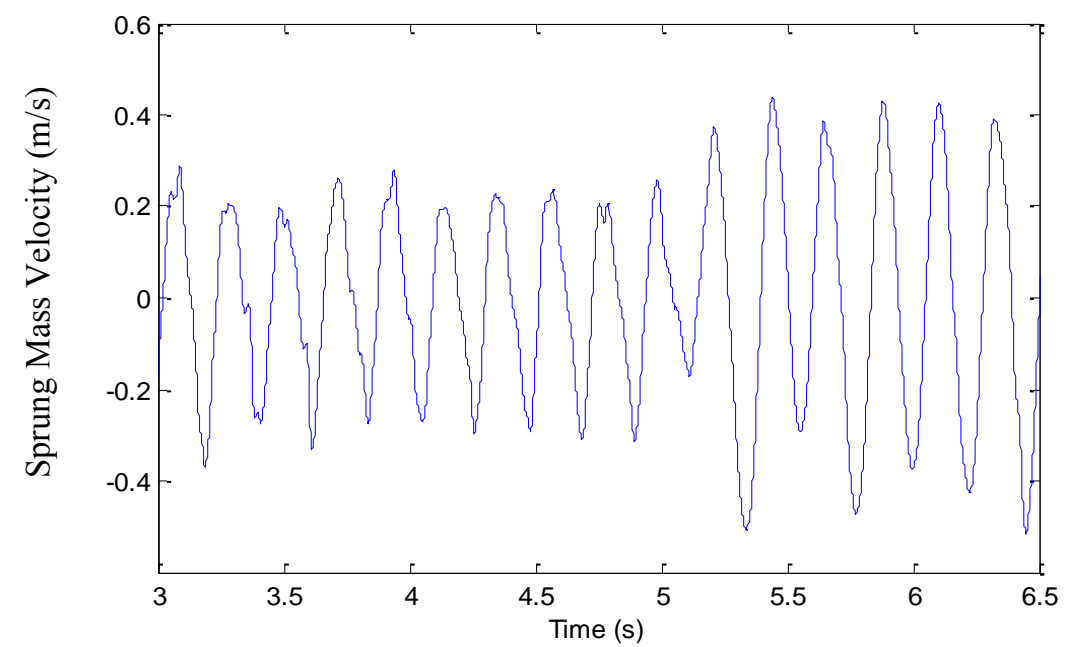

(b)

Figure 21. SMC result in case of (a) $\varepsilon=0.007$, and (b) $\varepsilon=0.008$, respectively.

In ideal SMC, switching could occur at an infinitely high frequency, which is impossible in practical implementation [24]. The fact that the control is constant within a sampling interval and the switching frequency cannot exceed that of sampling lead to chattering. Chattering is a harmful phenomenon because it leads to low control accuracy, wearing of moving mechanical parts, and heat losses in power circuits. To prevent 
chattering, a control law is implemented by introducing $\varepsilon$ for the $1-\mathrm{kHz}$ sampling rate. After several design iteration, control parameters were selected as $w_{1}=300, w_{2}=0.1$, $\alpha=0.001$, and $\varepsilon=0.004$.

Figure 20 shows the performances with different values of $\varepsilon$. There is no significant difference from the value from $\varepsilon=0.002$ to $\varepsilon=0.006$. Figure 20 (b) is generated by the use of same $\varepsilon$ as used in the Figure 24. However, Figure 20 (b) includes only the experimental result. As shown in figure 21, the performance begins to be distinctively degraded from $\varepsilon=0.007$.

Figure 22 shows significantly degraded performance due to inappropriate value of $\varepsilon$. When $\varepsilon$ reaches to the value of $\varepsilon=0.009$, disturbance attenuation performance is significantly degraded.

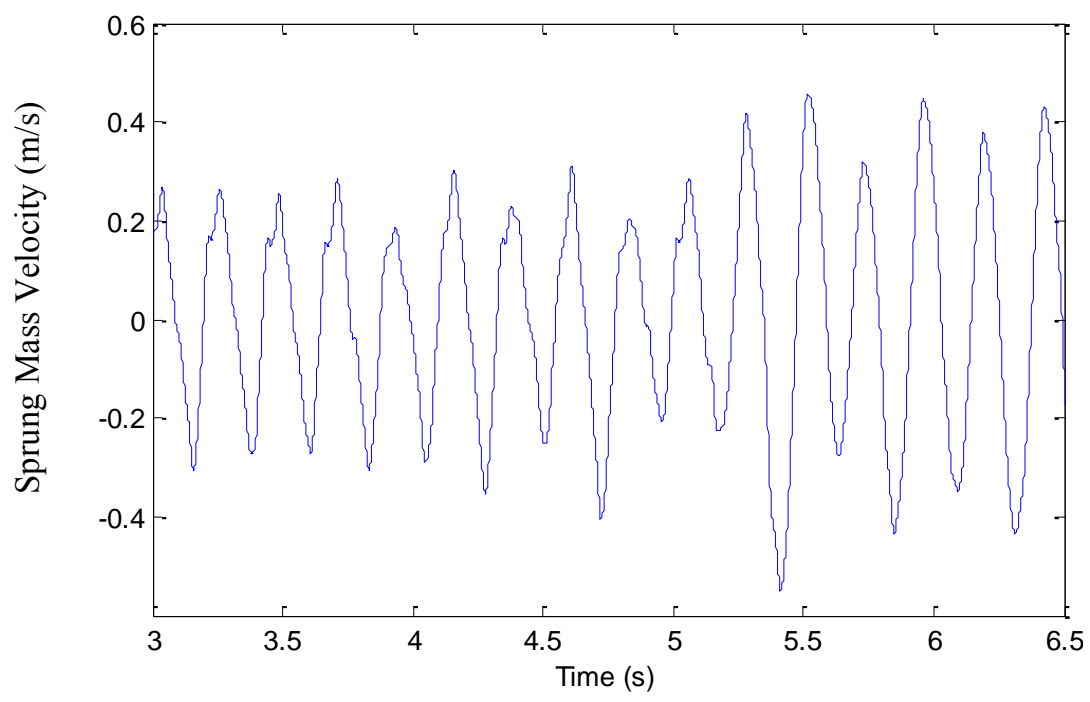

Figure 22. SMC result in case of $\varepsilon=0.009$. 
Figure 23 shows the state trajectory and sliding surface. The sliding surface has a large slope, i.e. $-\frac{w_{1}}{w_{2}}=-3000$. Switching between the two control laws occurs whenever the state trajectory goes across this sliding surface $(s>0$ in the right hand side of sliding surface and $s<0$ in the left hand side of sliding surface). The state trajectory forms two ellipses i.e. ellipse 1 and ellipse 2 . The ellipse 1 is the trajectory when the $\mathrm{SMC}$ is turned on, and the ellipse 2 is the one without SMC. Initially the sprung mass is under closed-loop control, and then the controller is turned off. Therefore, ellipse 1 is drawn first, and then the trajectory moves to the ellipse 2. The fact that each trajectory forms an ellipse implies that the sprung mass oscillates.

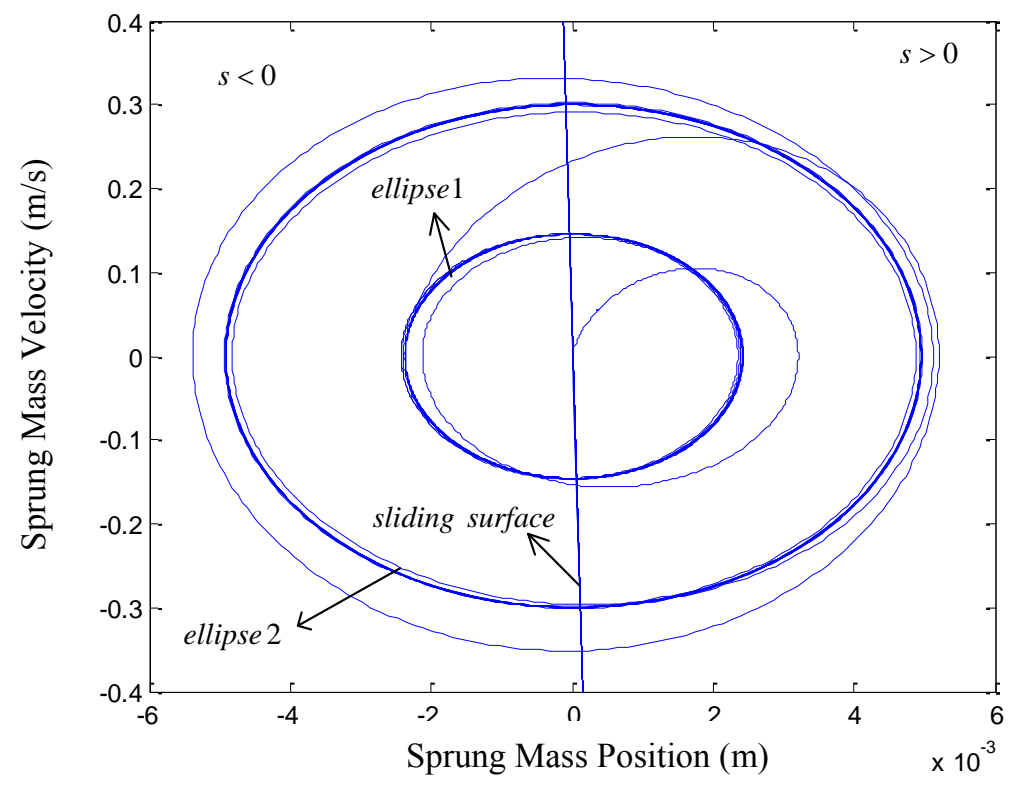

Figure 23. State trajectory and sliding surface. 
Figure 24 shows the experimental result and simulation result of the SMC. This controller is capable of attenuating the road disturbance approximately $55 \%$ and it is inferior to the other three controllers. It is mainly because the characteristics of $\varepsilon$. Even if $\varepsilon$ prevents the chattering phenomenon, it also degrades the performance because it intentionally generates a time delay before the control law is applied. During this time delay, the sprung mass moves without any regulation. This is a drawback of this chattering-proof method. Besides of the effect from $\varepsilon$, the fact that determining appropriate $\alpha$ is mostly done by design iteration is another reason of controller's inferior performance. The $\alpha$ is determined by keep tracking of the control input, $u$ from equation (30), and tested from the experiment. Aside from the applied method that prevents the chattering phenomenon and selecting $\alpha$, other approaches might enhance the performance of disturbance rejection in this research.

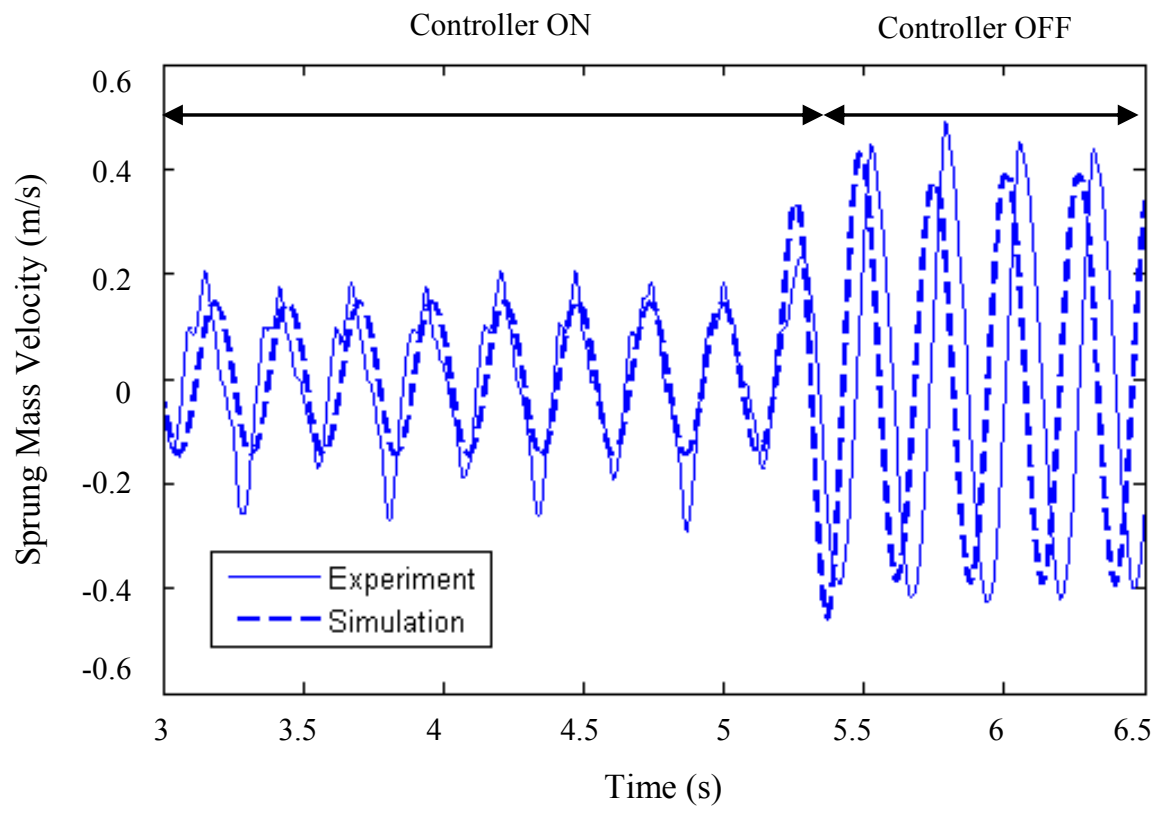

Figure 24. Experiment and simulation results of the SMC with $\varepsilon=0.004$. 
The figure 24 is the case of $\varepsilon=0.004$. This value is chosen among the values of $\varepsilon=0.002, \varepsilon=0.004$, and $\varepsilon=0.006$, which yield similar performances. It is because the value of $\varepsilon$ is compromised between the performance and the chattering-proof. Even though the smaller $\varepsilon$ results in the better performance, it is more likely inducing chattering phenomenon. Likewise, even if the larger $\varepsilon$ degrade the performance, it takes the system more away from the chattering phenomenon. 


\section{PERFORMANCE COMPARISONS}

In this section, the experimental results presented in the Section III are compared each other in terms of the disturbance attenuation performance. In this research, disturbance attenuation performance is defined as a magnitude ratio of the sprung mass velocity under the closed-loop control and the open-loop condition, respectively. The magnitude of the sprung mass velocity is defined as a peak-to-peak distance from the experimental results (Figure 10, 14, and 24). However, when the experimental results do not contain the constant peak value, peak value is assumed as an average value of those varying peak values.

The determination of the disturbance attenuation performance for the modified lead-lag control is shown in the Figure 25. The peak-to-peak value of the sprung mass velocity under the closed loop control ( lead-lag $_{\text {on }}$ ) is $0.24 \mathrm{~m} / \mathrm{s}$. The magnitude of the sprung mass velocity under the open loop condition $\left(\right.$ lead $\left.-\operatorname{lag}_{\text {off }}\right)$ is $1 \mathrm{~m} / \mathrm{s}$. Therefore, the disturbance attenuation performance is calculated as follows.

$$
P e r f_{\text {lead-lag }}=\left(1-\frac{\text { lead }- \text { lag }_{\text {on }}}{\text { lead }- \text { lag }_{\text {off }}}\right) \times 100=76 \%
$$

Figure 26 shows the disturbance attenuation performance of the LQ servo controller. The performance is calculated as follows.

$$
\operatorname{Perf}_{L Q}=\left(1-\frac{L Q_{o n}}{L Q_{o f f}}\right) \times 100=70 \%,
$$

where $L Q_{o n}=0.28 \mathrm{~m} / \mathrm{s}, L Q_{o f f}=0.89 \mathrm{~m} / \mathrm{s}$. 


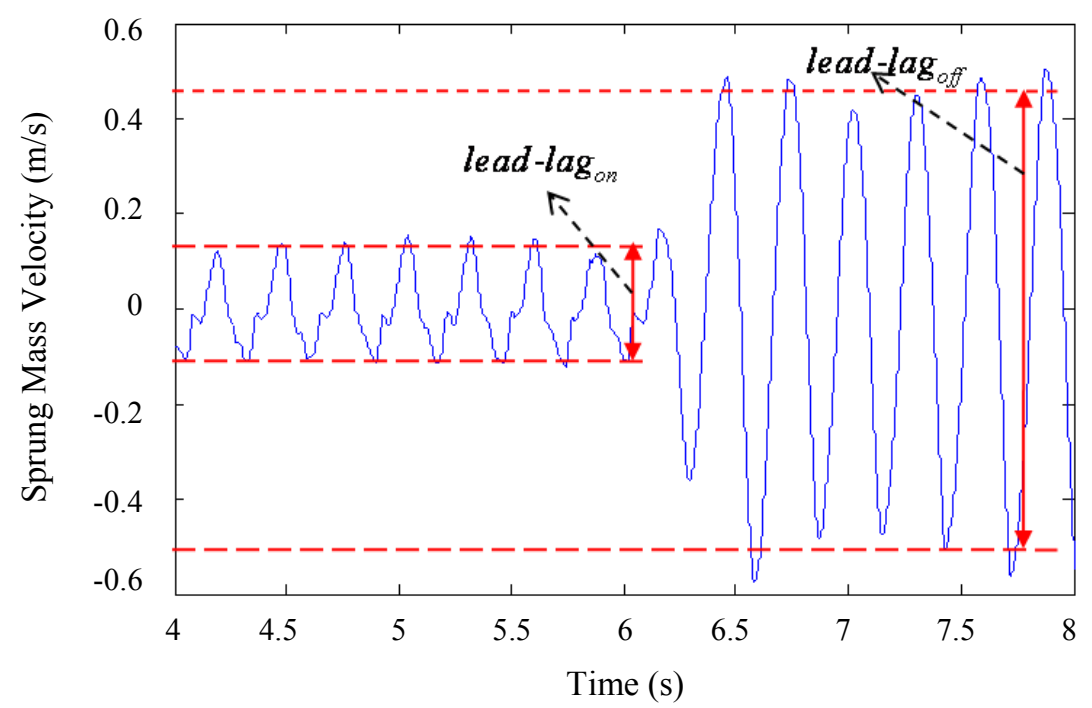

Figure 25. Disturbance attenuation performance of the modified lead-lag controller.

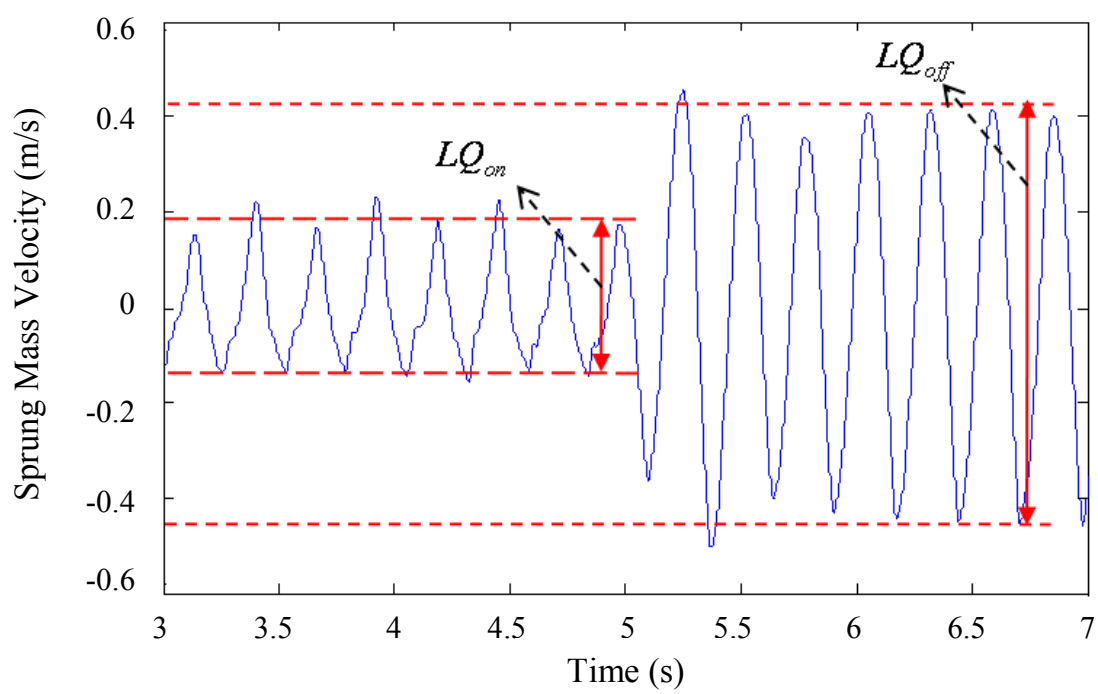

Figure 26. Disturbance attenuation performance of the LQ servo controller.

Figure 27 shows the disturbance attenuation performance of the fuzzy controller. The performance is calculated as follows. 


$$
\operatorname{Perf}_{f u z z y}=\left(1-\frac{f u z z y_{\text {on }}}{f u z z y_{\text {off }}}\right) \times 100=78 \% \text {, }
$$

where fuzzy $_{\text {on }}=0.22 \mathrm{~m} / \mathrm{s}$, fuzzy $y_{\text {off }}=1 \mathrm{~m} / \mathrm{s}$.

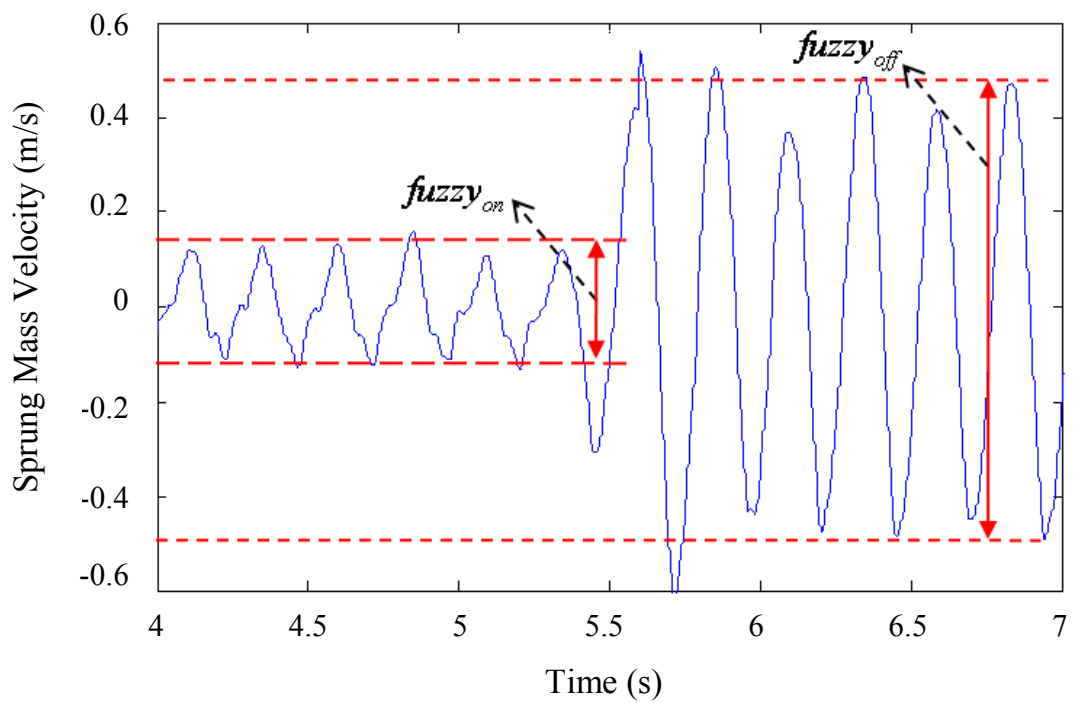

Figure 27. Disturbance attenuation performance of the fuzzy controller.

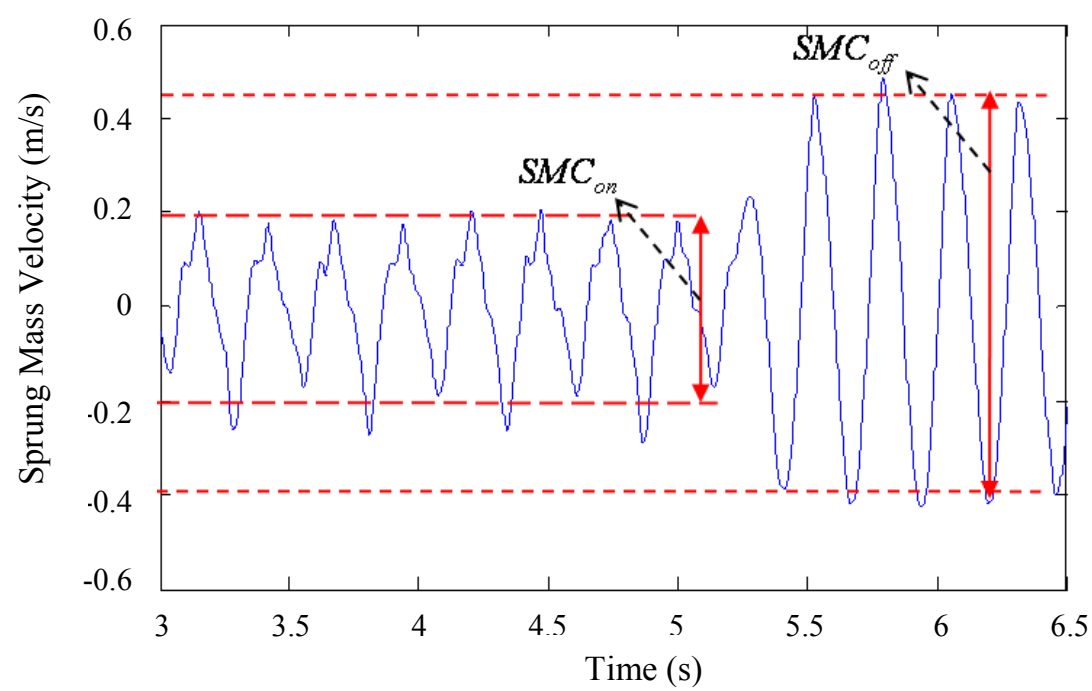

Figure 28. Disturbance attenuation performance of the SMC. 
Figure 28 shows the disturbance attenuation performance of the SMC. The performance is calculated as follows.

$$
\operatorname{Perf}_{S M C}=\left(1-\frac{S M C_{o n}}{S M C_{\text {off }}}\right) \times 100=55 \% \text {, }
$$

where $S M C_{o n}=0.4 \mathrm{~m} / \mathrm{s}, S M C_{\text {off }}=0.88 \mathrm{~m} / \mathrm{s}$.

The modified lead-lag and the fuzzy controller showed similar disturbance attenuation performances. The performance of the LQ servo controller was slightly degraded due to the Kalman filter. The performance of the SMC was inferior to other controllers due to the implemented chattering-proof algorithm that directly degrade the performance. 


\section{CONCLUSIONS}

Modified lead-lag, LQ servo, fuzzy, and SMCs were designed and implemented to attenuate modeled road disturbance in an active-suspension system with an LBPMM as a quarter-car test bed. In terms of the disturbance attenuation performance, the modified lead-lag and the fuzzy controller showed superior experimental results to the other two controllers.

The modified lead-lag controller consisted of two lag controllers and one lead controller. Each lead and lag controller was designed to satisfy its own control objectives. Finally, lead and lag controllers were fine-tuned to determine their exact corner frequencies. Selecting its design parameters did not require too many design iterations to satisfy the control objectives. Also, modified lead-lag control required no LVDT. Therefore, the modified lead-lag controller was a fairly acceptable in this research.

In the case of the LQ servo, performance of disturbance rejection was slightly inferior to the modified lead-lag and the fuzzy controllers. The reason is that the estimator could not perfectly generate the estimated state because the noise and the disturbance were not white Gaussian. Moreover, an additional sensor (the LVDT) was required in this control method. Therefore, both performance- and cost-effectivenesswise, the LQ servo was not suitable for this application.

When it comes to the fuzzy controller, it turned out to be the most suitable control methodology for this active-suspension application. It is because its asymmetric membership functions allowed the LBPMM to generate the most suitable control force to mitigate the error characteristics due to non-idealities of the test bed. However, this 
fuzzy controller was difficult to design since it has infinitely many design parameters such as selecting the domains for the fuzzification and defuzzyfication.

Unlikely to the other three controllers, SMC was designed to control the position rather than the velocity of the sprung mass for the convenient construction of the sliding surface. In this point of view, it is not fair for this controller to be compared to other three controllers. To avoid the chattering phenomenon, a small time delay was placed when the state variable goes across the sliding surface before the control laws were applied. Even though this algorithm was implementable without much difficulty, it directly degraded the performance. This is the primary reason why this controller showed the most inferior performance.

In summary, an LBPMM, a unique tubular linear motor, was successfully employed as the key actuator in active suspension. When it comes to the control, the fuzzy controller was the most suitable controller since the design parameters for the asymmetric membership functions were finalized with the results from the modified lead-lag and LQ servo control. The modified lead-lag control was fairly acceptable. However, LQ servo was not an appropriate control methodology for this application. Additionally, SMC was constructed for the position control with an intuitive method that prevents the chattering phenomenon. Its performance was the most inferior to the other three controllers. 


\section{REFERENCES}

[1] J. Allen, Design of Active Suspension Control Based Upon Use of Tubular Linear Motor and Quarter-Car Model, Master's Thesis, Texas A\&M University, August 2008.

[2] H. Peng, R. Stratharn, and A. Ulsoy, "A Novel Active Suspension Design Technique-Simulation and Experimental Results," in Proc. 1997 American Control Conference, June 1997, pp. 709-713.

[3] C. Tang and T. Zhang, "The Research on Control Algorithms of Vehicle Active Suspension System," in Proc. IEEE International Conference on Vehicular Electronics and Safety, October 2005, pp. 320-325.

[4] Y. M. Sam, M. R. H. A. Ghani, and N. Ahmad, "LQR Controller for Active Car Suspension," in Proc. of TENCON 2000, September 2000, pp. 441-444.

[5] C. Lauwerys, J. Swevers, and P. Sas, "Design and Experimental Validation of a Linear Robust Controller for an Active Suspension of a Quarter-Car," in Proc. 2004 American Control Conference, July 2004, pp. 1481-1486.

[6] J. Wang, A. C. Zolas, and D. A. Wilson, “Active Suspension: A Reduced-Order $H_{\infty}$ Control Design Study," in Proc. 2007 Mediterranean Conference on Control and Automation, July 2007, pp. 132-140.

[7] C. Abbas, T. Rahaijaona, and H. Noura, "Sliding-mode Control Applied to Active Suspension Using Nonlinear Full Vehicle and Actuator Dynamics," in Proc. IEEE conference on Decision \& Control, December 2006 pp. 3597-3602. 
[8] Y. Jin, D. Yu, and X. Song, “An Integrated-Error-Based Adaptive Neuron Control and its Application to Vehicle Suspension Systems," in Proc. IEEE International Conference on Control and Automation, May 2007, pp. 564-569.

[9] F. Kou and Z. Fang, "An Experimental Investigation into the Design of Vehicle Fuzzy Active Suspension," in Proc. IEEE International Conference on Automation and Logistics, August 2007, pp. 959-963.

[10] A. Alleyne and J. Hedrick, "Nonlinear Adaptive Control of Active Suspension," IEEE Transactions on Control Systems Technology, vol. 3, no. 1, pp. 91-101, March 1995.

[11] R. Rajamani, “Adaptive Observers for Active Automotive Suspensions: Theory and Experiment," IEEE Transactions on Control Systems Technology, vol. 3, no. 1, pp. 86-93, March 1995.

[12] W.-J. Kim and B. Murphy, "Development of a Novel Direct-Drive Tubular Linear Brushless Permanent-Magnet Motor," International Journal of Control, Automation, and System, vol. 2, no. 3, pp. 279-288, September 2004.

[13] R. Williams, "Control of a Low Frequency Active Suspension," in Proc. International Conference on Control, vol. 1, pp. 338-343, March 1994.

[14] M. Hoque, M. Yakasaki, Y. Ishino, and T. Mizuno, "Design of a Mode-Based Controller for 3-DOF Vibration Isolation System," in Proc. of IEEE International Conference on Robotics, Automation and Mechatronics, December 2004, pp. 478483. 
[15] H. Gao, J. Lam, and C. Wang, "Multi-Objective Control of Vehicle Active Suspension Systems via Load-Dependent Controllers," Journal of Sound and Vibration, vol. 290, pp. 654-675, March 2006.

[16] N. Yagiz, I. Yuksek, and S. Sivrioglu, "Robust Control of Active Suspensions for a Full Vehicle Model Using Sliding-mode Control," International Journal of the Japan Society of Mechanical Engineers, vol. 43, no. 2, pp. 253-258, July 2000.

[17] Y. Suda and T. Shiba, "A New Hybrid Suspension System with Active Control and Energy Regeneration,” International Journal of Vehicle Mechanics and Mobility, vol. 25, pp. 641-654, January 1996.

[18] J. Concha and A. Cipriano, "A Design Method for Stable Fuzzy LQR Controllers," in Proc. Sixth IEEE Internationsl Conference, vol. 1, pp. 271-276, July 1997.

[19] M. Gobbi, F. Levi, and G. Mastinu, "Multi-Objective Stochastic Optimization of the Suspension System of Road Vehicles," Journal of Sound and Vibration, vol. 298, pp. 1055-1072, 2006.

[20] G. Stein, "Respect the Unstable," IEEE Control Systems Magazine, vol. 23, no. 4, p. 12-25, August 2003.

[21] J. Gere, Mechanics of Materials, location: Brooks/Cole, p. 899, 2001

[22] B. Anderson and J. Moore, Optimal Control: Linear Quadratic Methods, location: Prentice-Hall, p. 74, 1989.

[23] K. Passino and S. Yurkovich, Fuzzy Control, location: Addison-Weslsy, p. 42, 1999. 
[24] C. Edward and S. Spurgeon, Sliding-mode Control: Theory and Applications, location: Taylor \& Francis, p. 7, 1998. 


\section{APPENDIX A: SIMULINK BLOCK DIAGRAMS}

A.1 Simulink block diagram for real-time control: Modified lead-lag control

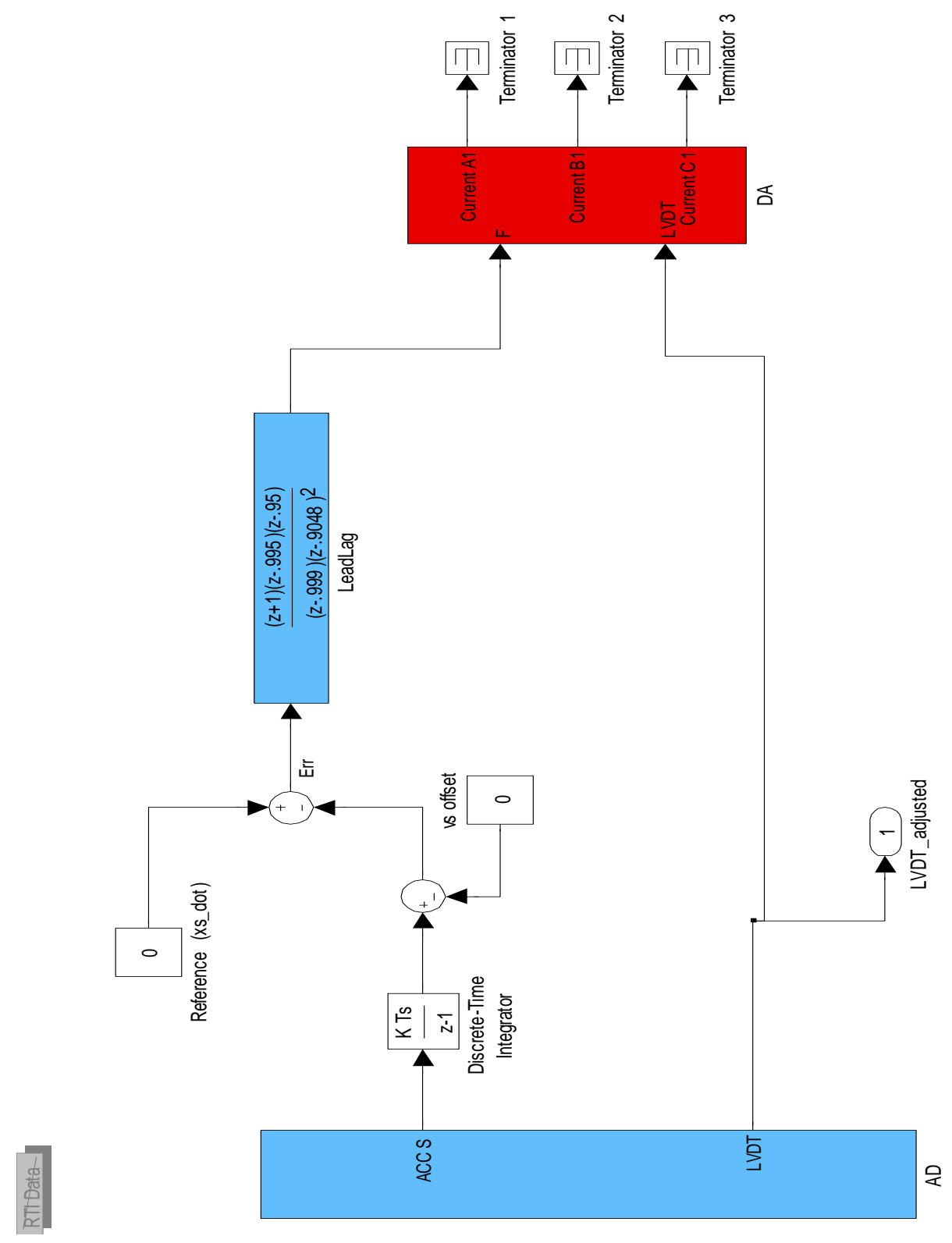


A.2 Simulink block diagram for real-time control: LQ Servo control

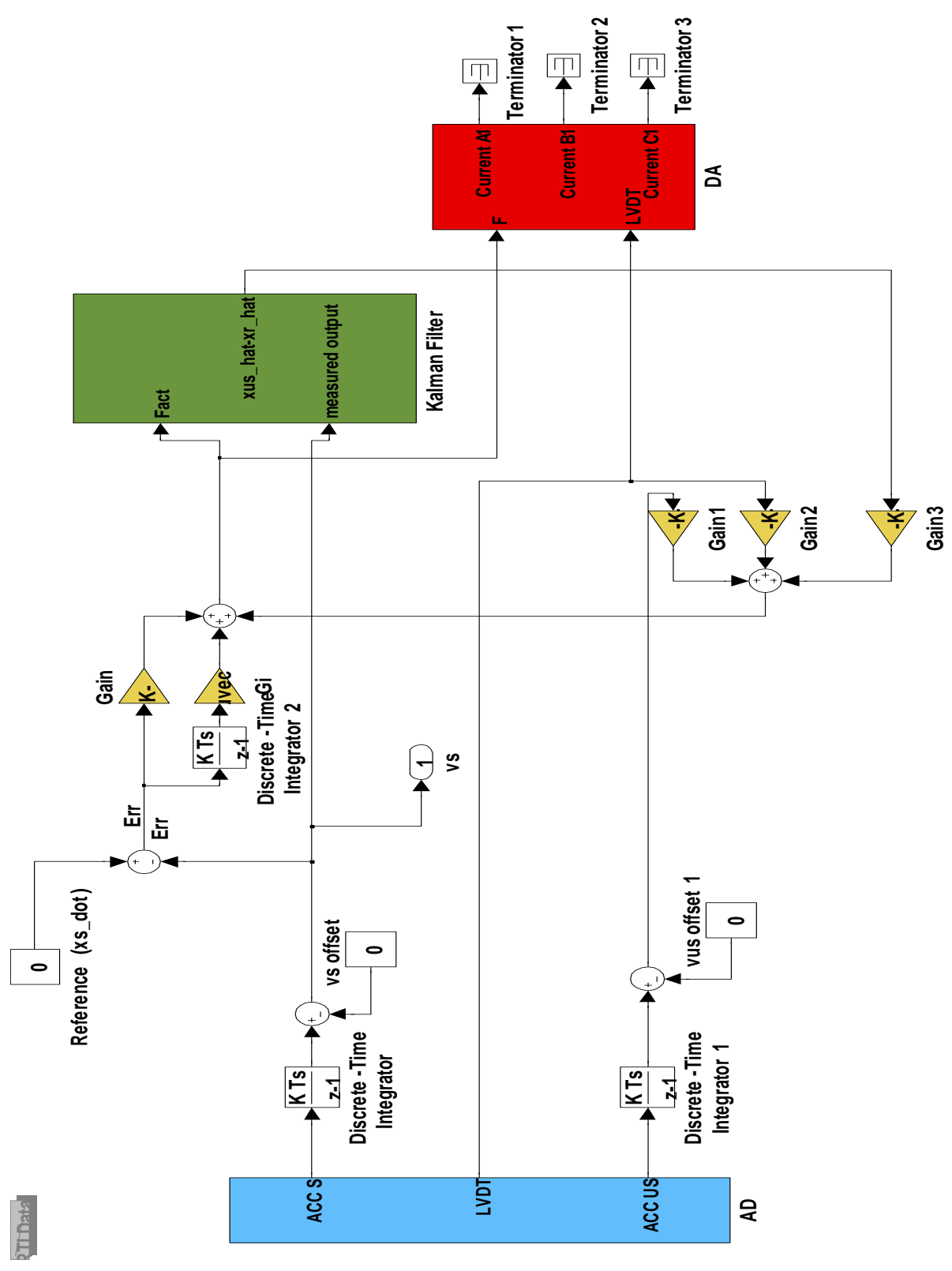




\section{A.3 LQ Servo control: Kalman Filter}

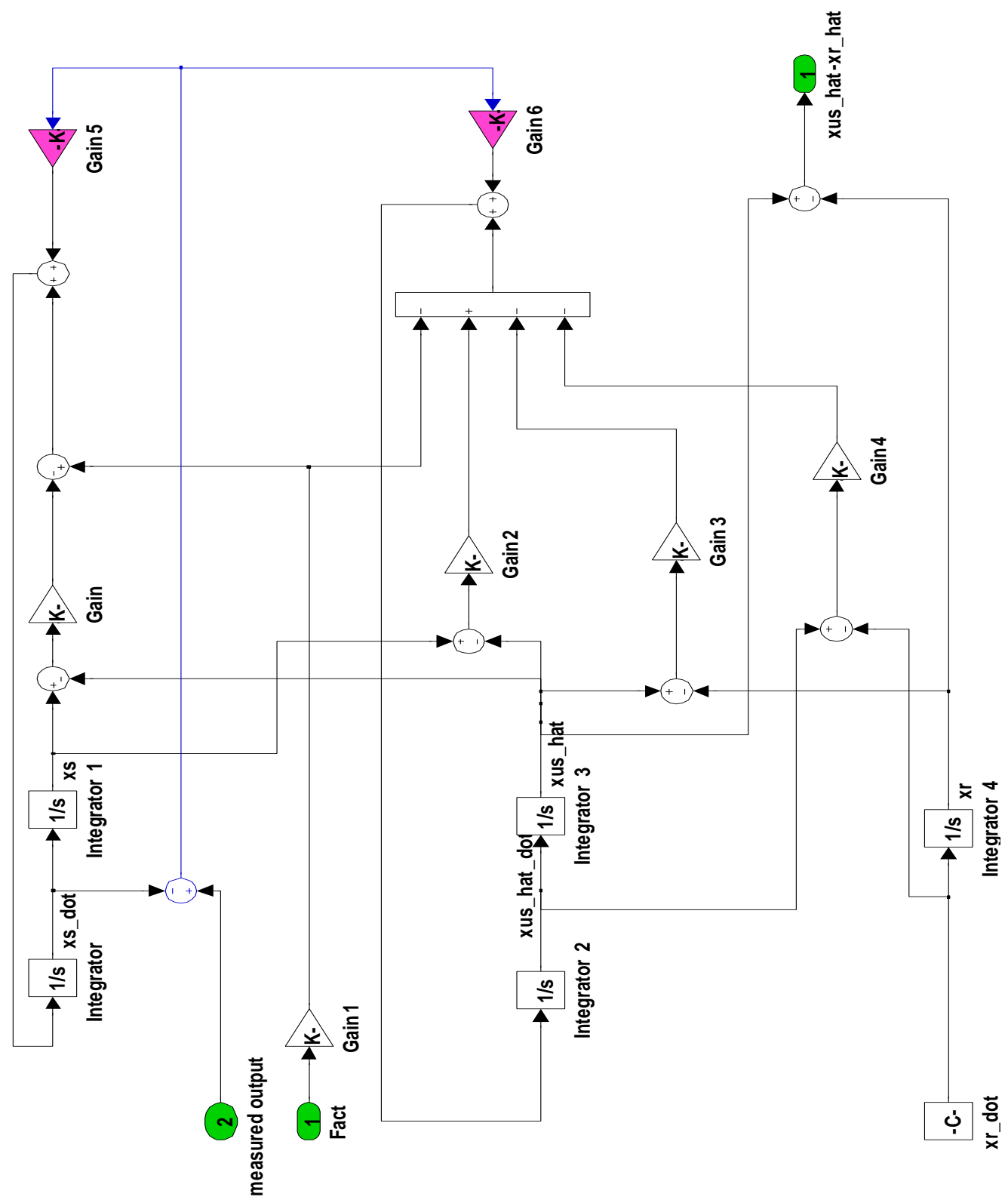


A.4 Simulink block diagram for real-time control: Fuzzy control

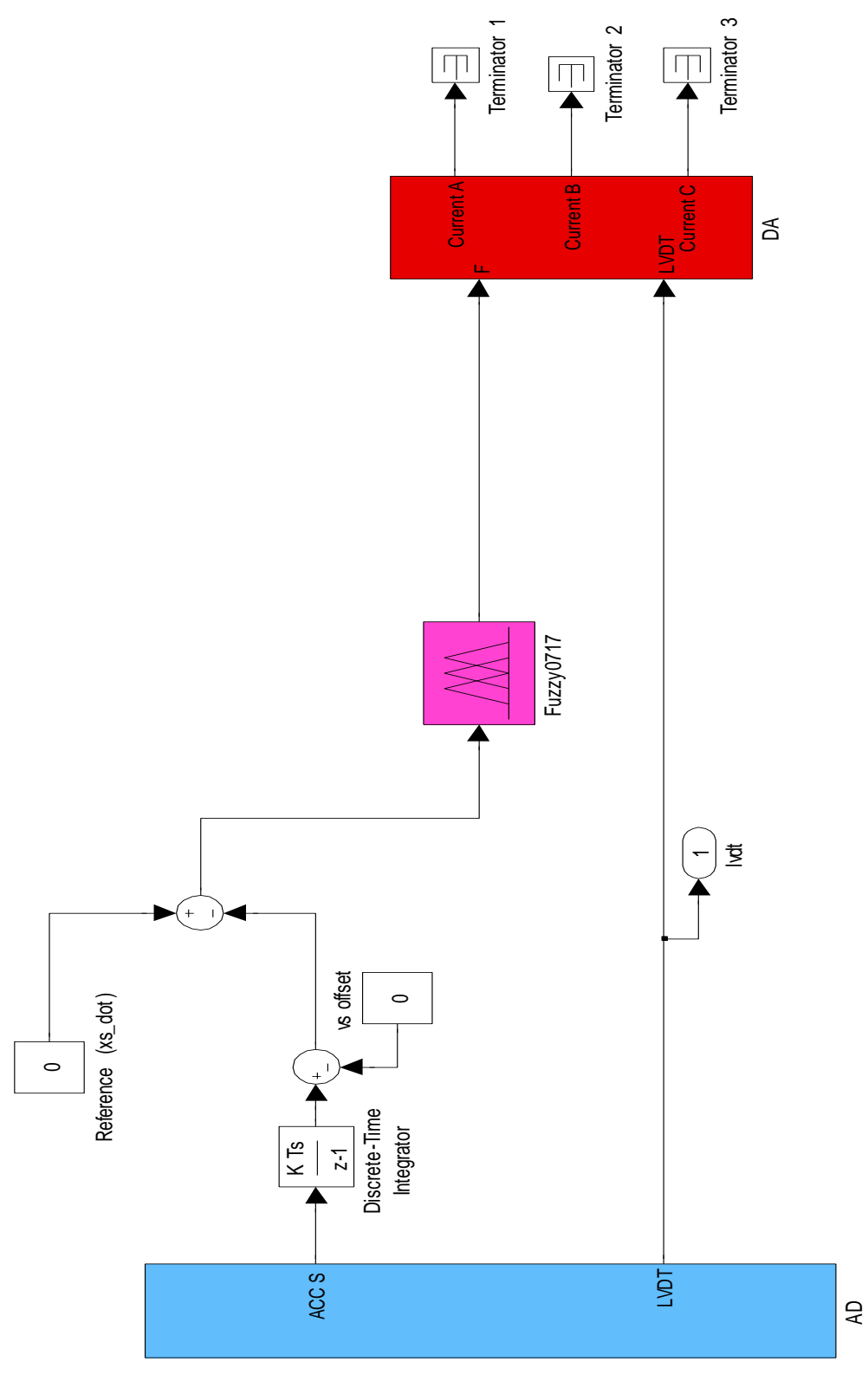


A.5 Implementation of the fuzzy controller block

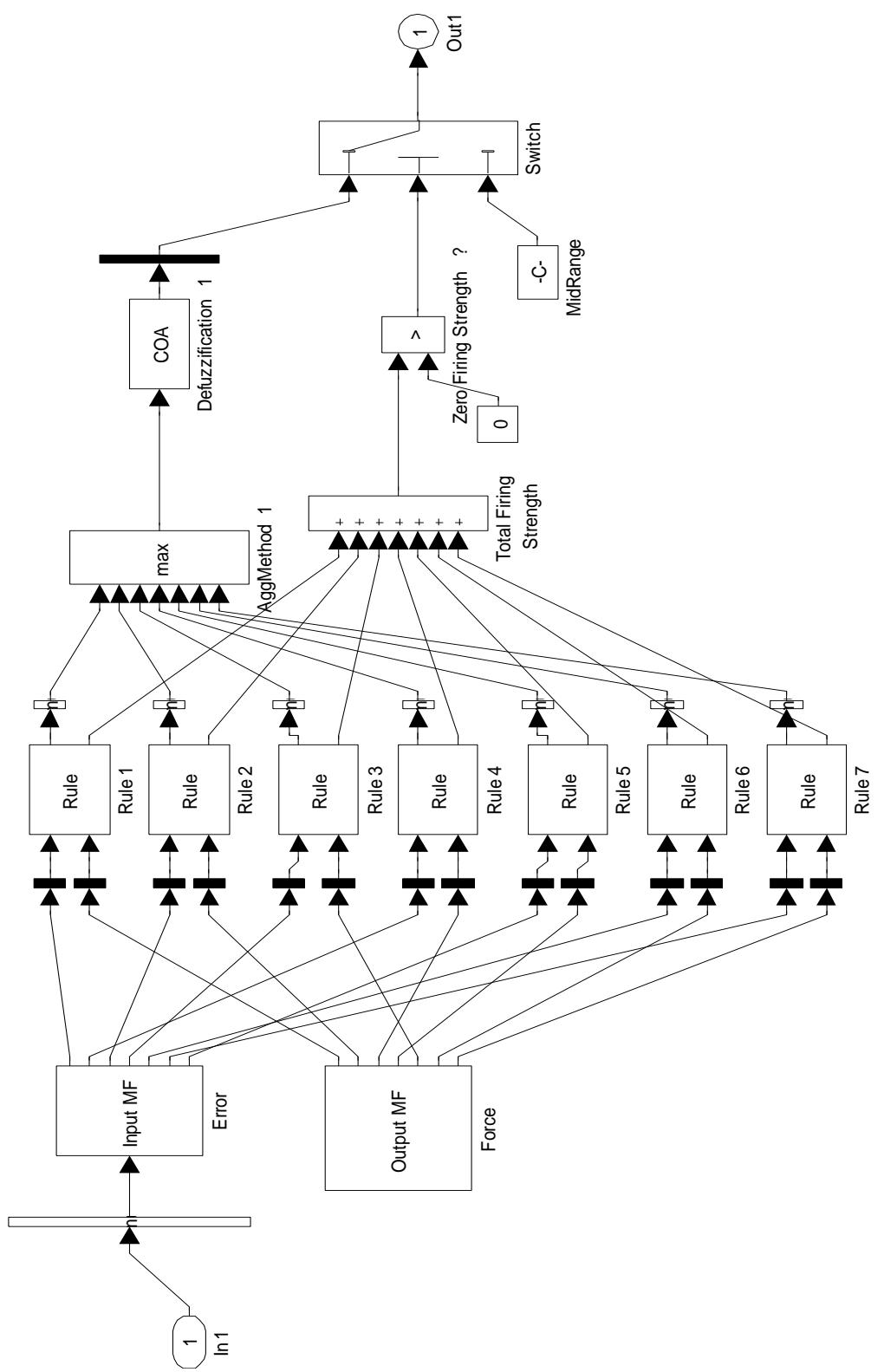


A.6 Simulink block diagram for real-time control: Sliding-mode control

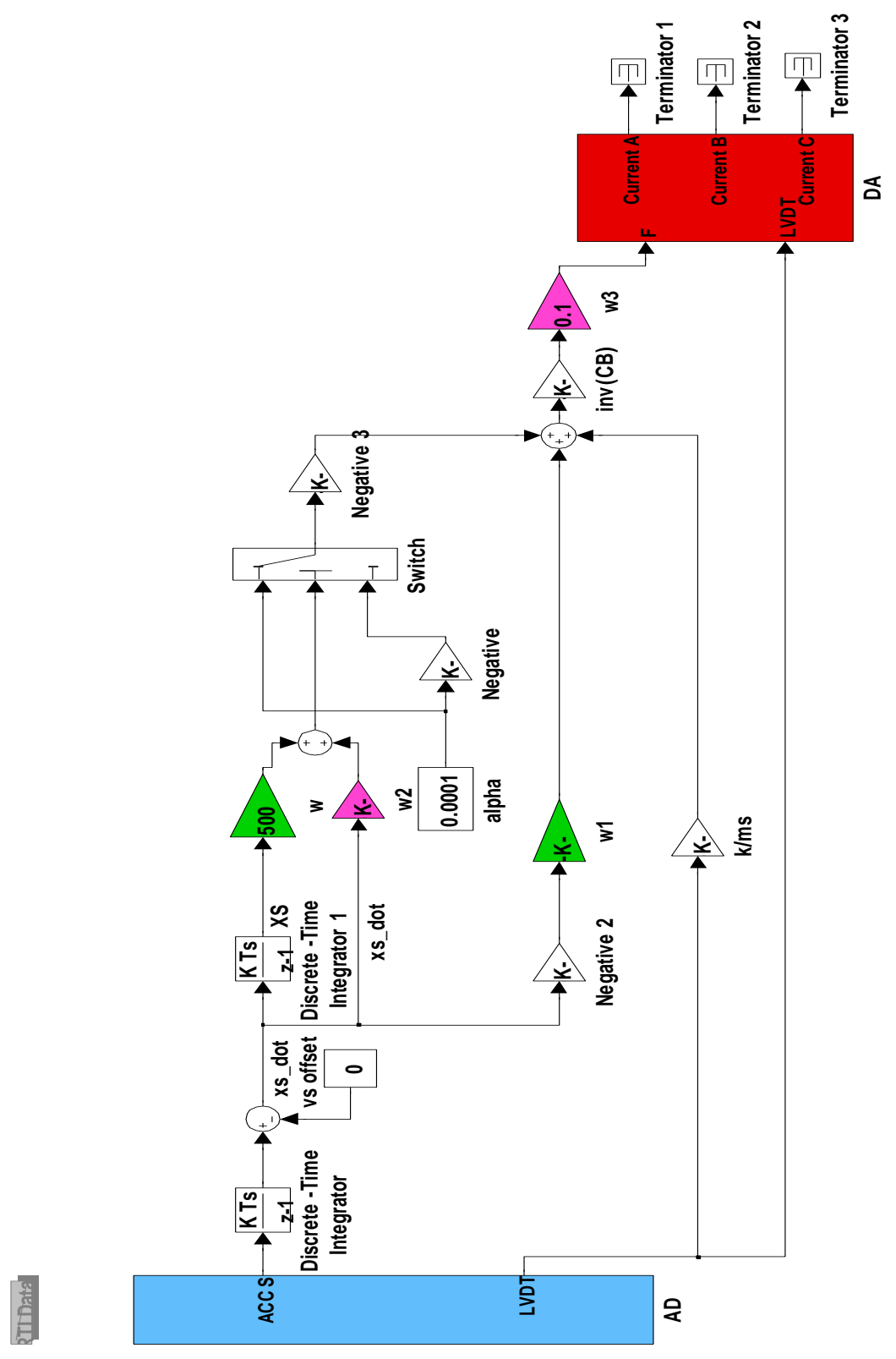


A.7 Analog-to-Digital input port

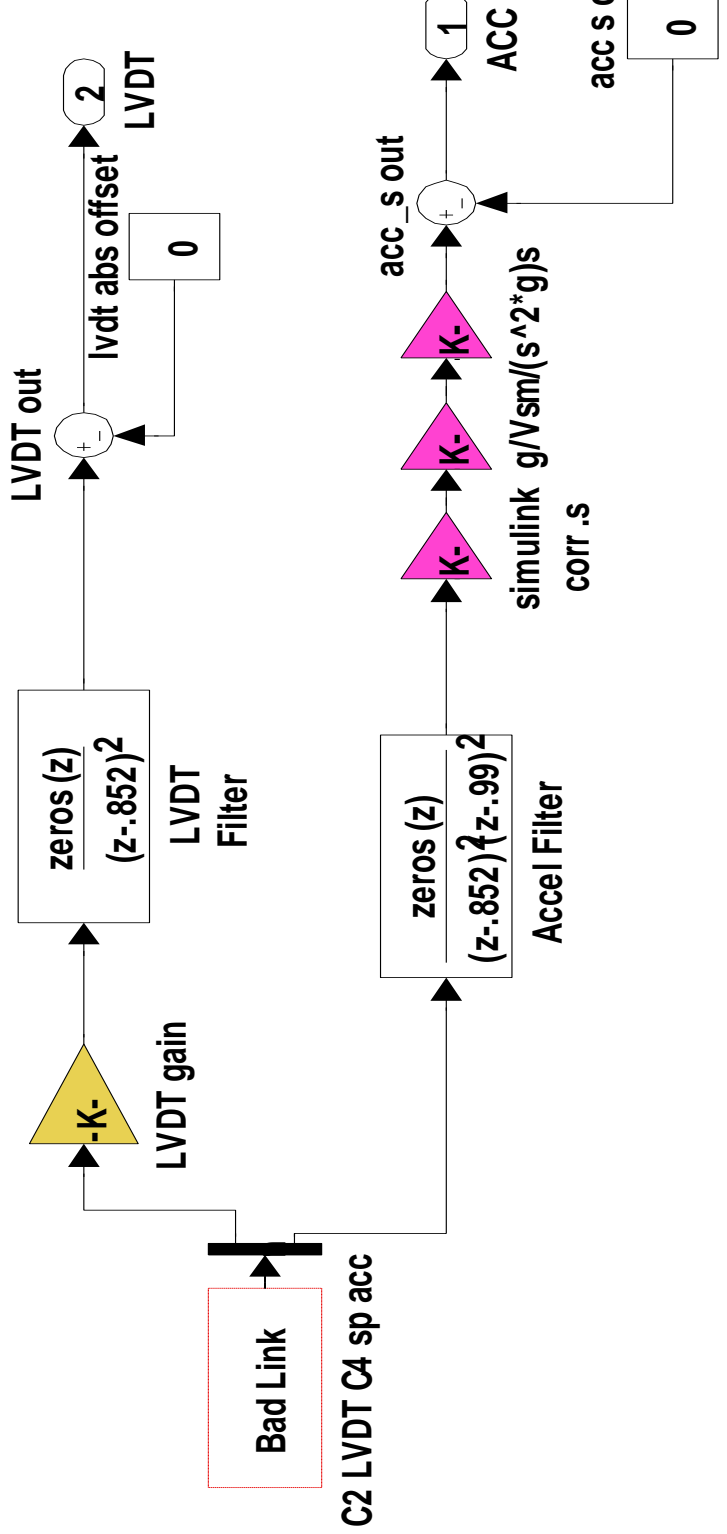


A.8 Digital-to-Analogue output port

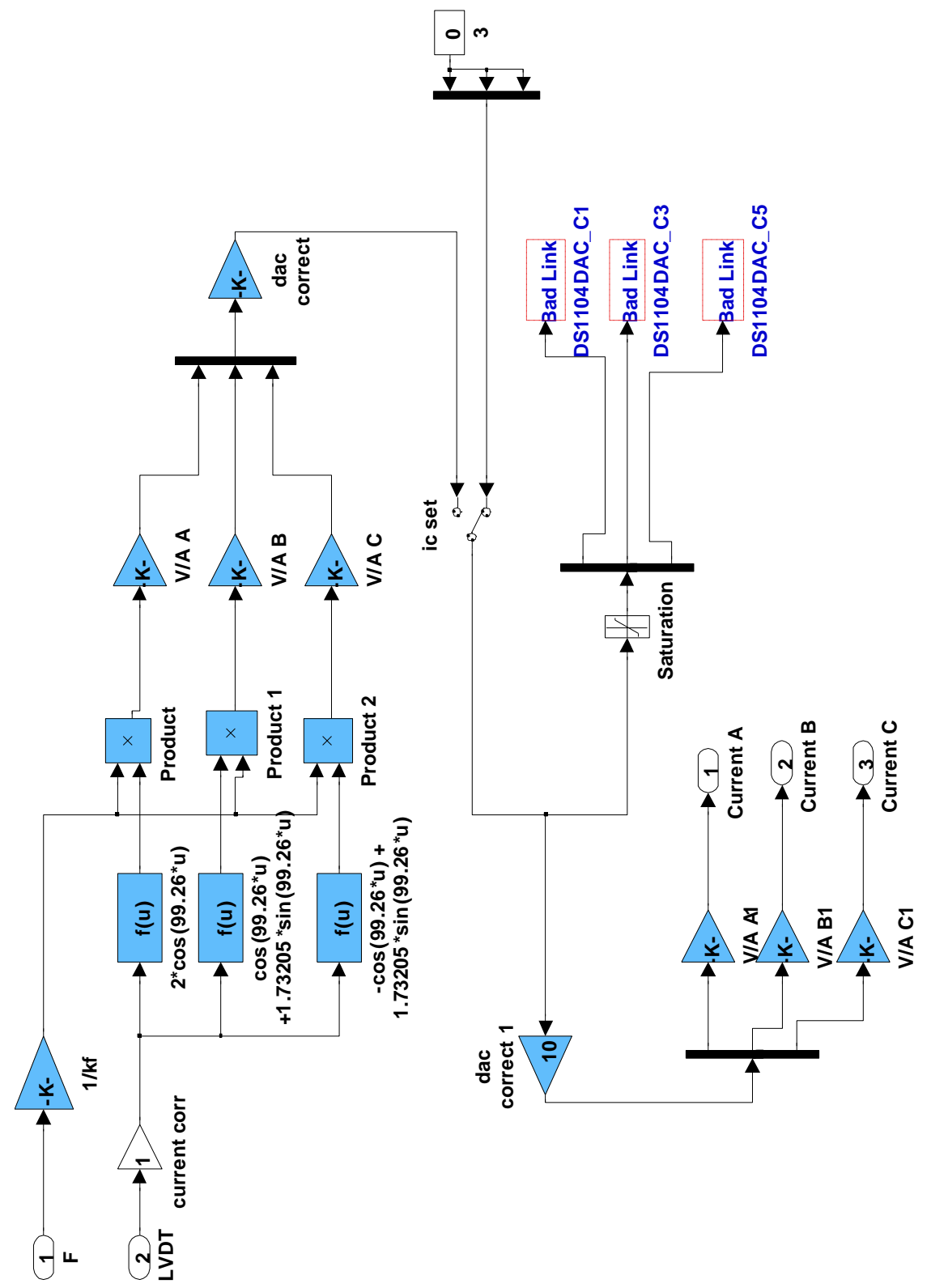




\section{APPENDIX B: CONTROL DESK SCREEN SHOTS}

B.1 Control Desk screen shot: Modified lead-lag control

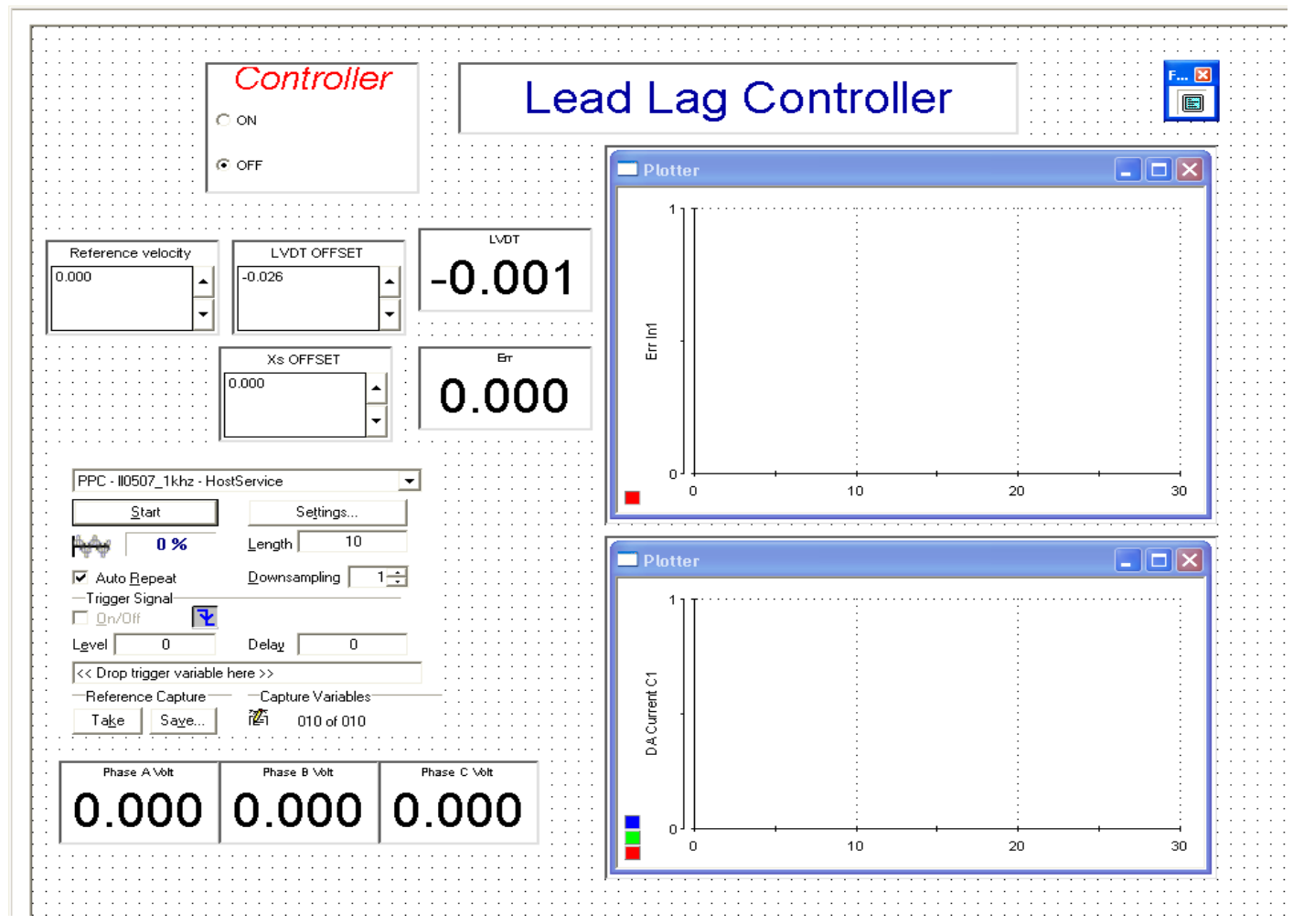


B.2 Control Desk screen shot: LQ Servo control

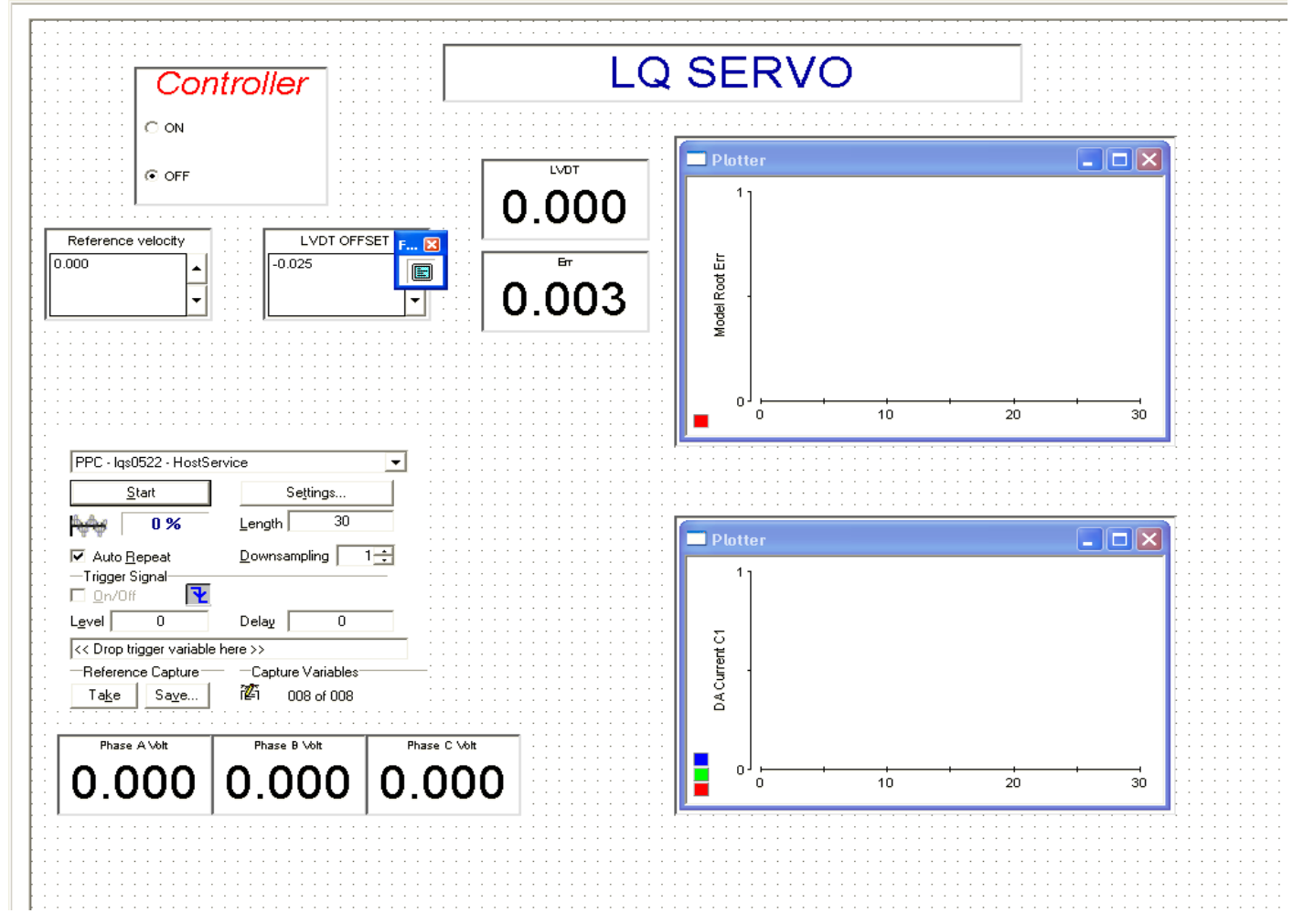


B.3 Control Desk screen shot: Fuzzy control

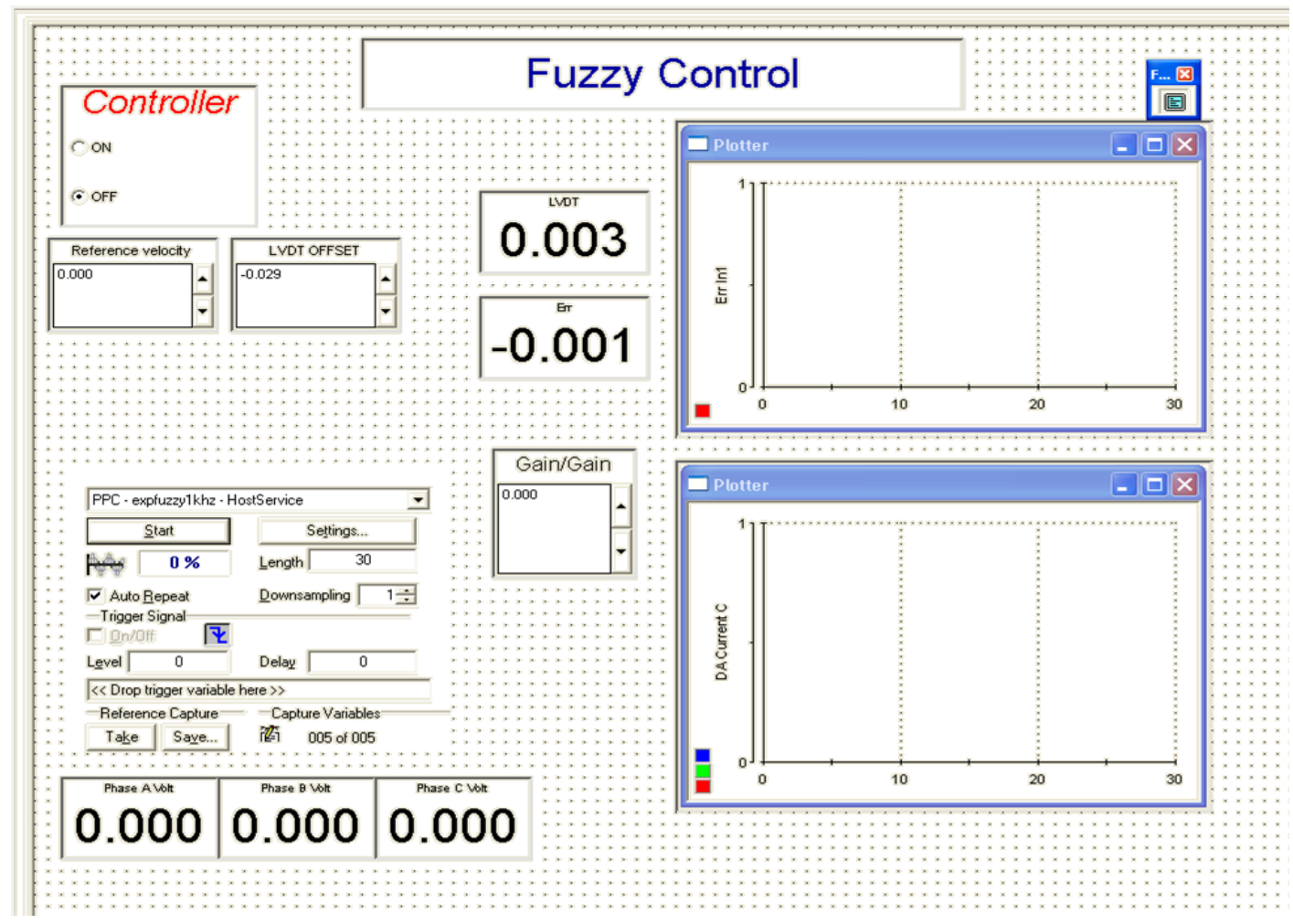


B.4 Control Desk screen shot: Sliding-mode control

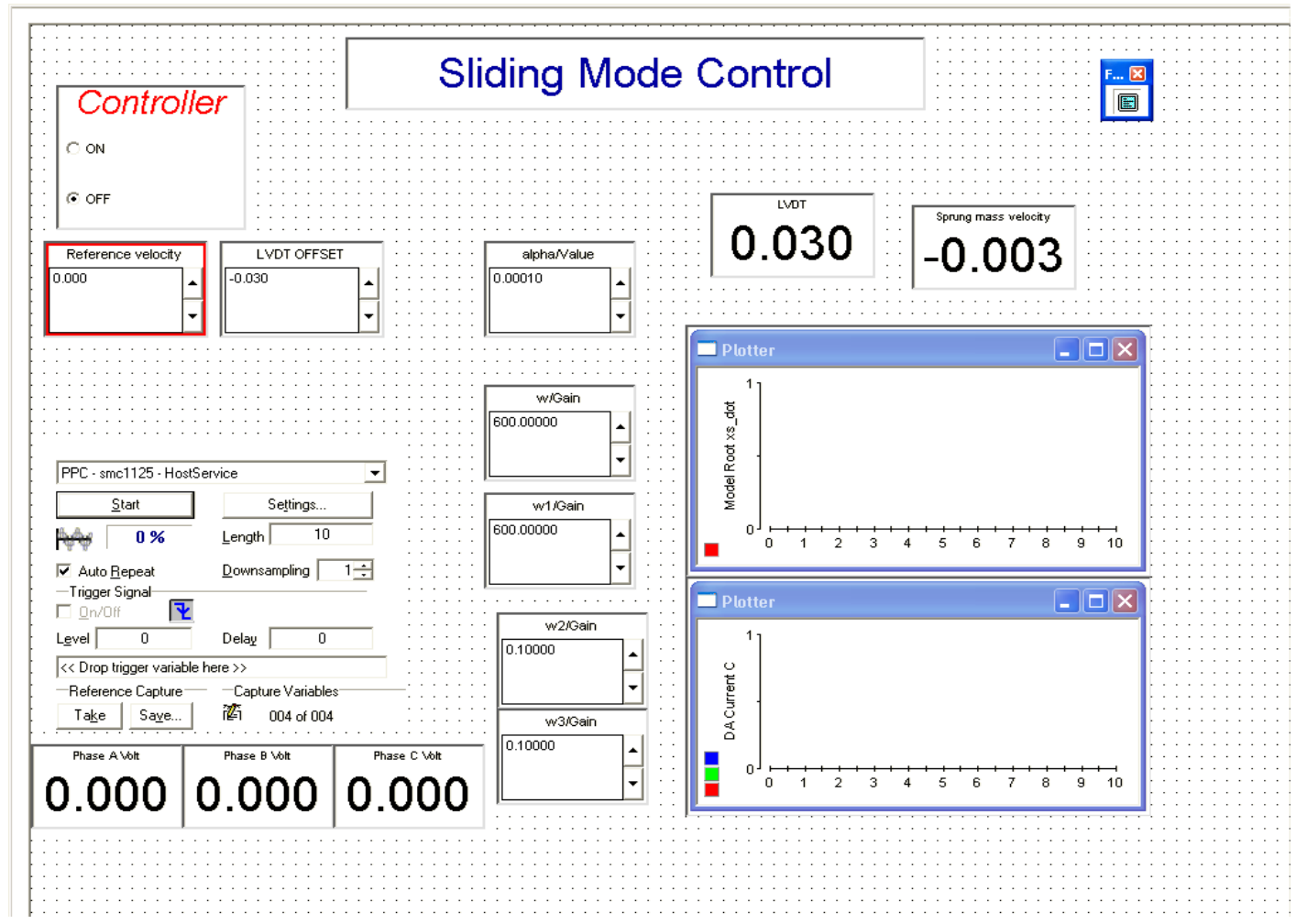




\section{VITA}

Seungho Lee received his Bachelor of Science degree in Computer Science and Mechanical Engineering from Yonsei University in 2001 and 2005, respectively. Since 2007, he has been working towards the M.S. degree in the Mechanical Engineering Department at Texas A\&M University, College Station. He joined the Precision Mechatronics and Nanotechnology Laboratory in January 2008. His research interests include analysis and design of control systems.

Name: $\quad$ Seungho Lee

Address: $\quad$ Mail box J05, Texas A\&M University, Department of Mechanical Engineering, 3123 TAMU, College Station, TX 77843-3123

Email Address: wckorealsh@gmail.com

Education: $\quad$ B.S., Computer Science, Yonsei University, 2001

B.S., Mechanical Engineering, Yonsei University, 2005 